\title{
Bifurcations of cubic homoclinic tangencies in two-dimensional symplectic maps *
}

\author{
M. Gonchenko ${ }^{1}$, S.V. Gonchenko² and I. Ovsyannikov ${ }^{3}$ \\ ${ }^{1}$ Departament de Mathemàtiques i Informàtica, Universitat de Barcelona, Spain \\ ${ }^{2}$ N.I. Lobachevsky Nizhny Novgorod University, Russia \\ ${ }^{3}$ Fachbereich Mathematik und Informatik, Universität Bremen, Germany \\ gonchenko@ub.edu, gonchenko@pochta.ru, ivan.i.ovsyannikov@gmail.com
}

\begin{abstract}
We study bifurcations of cubic homoclinic tangencies in two-dimensional symplectic maps. We distinguish two types of cubic homoclinic tangencies, and each type gives different first return maps derived to diverse conservative cubic Hénon maps with quite different bifurcation diagrams. In this way, we establish the structure of bifurcations of periodic orbits in two parameter general unfoldings generalizing to the conservative case the results previously obtained for the dissipative case. We also consider the problem of 1:4 resonance for the conservative cubic Hénon maps.
\end{abstract}

\section{Introduction and main results}

Homoclinic tangencies, i.e. tangencies between stable and unstable invariant manifolds of the same saddle periodic orbit, play a very important role in the theory of dynamical chaos. Starting with the works by Smale and - Shilnikov [Sma65, Shi67] (and in the ideological sense as far back as with the memoirs by Poincaré and Birkhoff [Poi90, "Poi99, Bir35]), the existence of transversal homoclinic intersections is considered as the universal criterium of the complexity of dynamical systems. At the same time, the presence of non-transversal homoclinic orbits (homoclinic tangencies) indicates an extraordinary richness of bifurcations of such systems and, what is very important, the principal impossibility of providing of a complete description of bifurcations of such systems within framework of finite parameter families, GST93, GST99, Kal00, DN05, GST07. Therefore, when studying homoclinic bifurcations, the main problems are related to the analysis of their principal bifurcations and characteristic properties of dynamics as a whole.

One of the important properties of homoclinic tangencies is that they can freely produce homoclinic and heteroclinic tangencies of higher orders [GST93, GST96, GST99, GST07]. In particular, in a two parameter family which unfolds generally a quadratic homoclinic tangency, cubic homoclinic tangencies appear unavoidably. This means that the study of bifurcations of cubic homoclinic tangencies itself becomes an important problem. Moreover, cubic homoclinic tangencies can play an independent role when studying global bifurcations in many dynamical models. For example, such tangencies appear naturally in the problem on periodically perturbed two-dimensional flows with a homoclinic figure-eight of a saddle equilibrium GSV13, where the corresponding bifurcation points of codimension two give rise to the curves of quadratic homoclinic tangencies forming (non-smooth) boundaries of homoclinic zones. Moreover, bifurcations of cubic tangencies lead to the appearance of specific windows of stability (i.e. parameter domains corre-

*This work has been partially supported by the Russian Scientific Foundation Grant 14-41-00044, and the Spanish MINECO-FEDER Grant MTM2015-65717-P. The numerical experiments in Section 3 have been carried out within the RSciF-grant (project No.14-1200811). Author MG has been partially supported by Juan de la Cierva-Formación Fellowship FJCI-2014-21229. MG warmly thanks the Department of Mathematics of Uppsala University for their hospitality and support; during her stay at Uppsala University, MG was also partially supported by the Knut and Alice Wallenberg Foundation grant 2013-0315. Author SG has been partially supported by the grant of RFBR No. 16-01-00324 and 14-01-00344. Author IO has been supported by the project M2 (Systematic multi-scale modelling and analysis for geophysical flow) of the Collaborative Research Centre TRR 181 "Energy Transfer in Atmosphere and Ocean" funded by the German Research Foundation. 

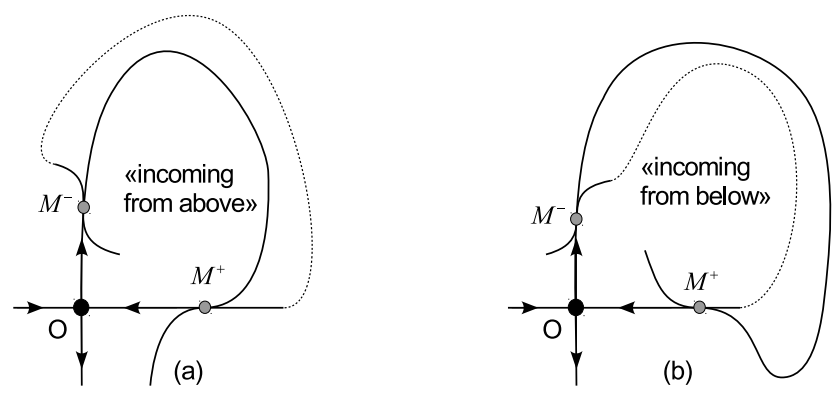

Figure 1: Two types of cubic homoclinic tangencies: (a) incoming from above and (b) incoming from below.

sponding to the existence of stable periodic orbits) which are well observable during numerical explorations, GSV13, unlike the windows produced by quadratic homoclinic tangencies.

In fact, bifurcations of cubic homoclinic tangencies were studied first in Gon85 for the sectionally dissipative case (when a saddle periodic orbit has eigenvalues $\lambda_{1}, \ldots, \lambda_{n}, \gamma$ such that $|\gamma|>1>|\lambda|=\max _{i}\left|\lambda_{i}\right|$ and $\sigma=|\lambda \gamma|<1$ ), see also GST96, GSV13, Tat92. Note that in Gon85 the two main cases were considered when the leading stable eigenvalue is real (i.e. $\left|\lambda_{1}\right|>\left|\lambda_{i}\right|, i=2, \ldots, n$, ) and the two leading eigenvalues are complex conjugate (i.e. $\lambda_{1,2}=$ $\rho e^{ \pm i \varphi}, \varphi \neq 0, \pi$, and $\left.\rho>\left|\lambda_{i}\right|, i=3, \ldots, n\right)$. The main attention in Gon85] was focused on the study of bifurcations of single-round periodic orbits, i.e. periodic orbits which pass a neighborhood of the homoclinic orbit only once.

We note that the study of such orbits can be reduced to an analysis of fixed points of the corresponding first return maps defined near some point of the homoclinic tangency. In the case of cubic tangencies, such maps can be brought to the maps close to the two-dimensional cubic Hénon maps of the form

$$
\mathbf{C}_{ \pm}^{J}: \bar{x}=y, \quad \bar{y}=M_{1}+M_{2} y-J x \pm y^{3}
$$

being $M_{1}$ and $M_{2}$ parameters and $J$ the Jacobian. Moreover, map $\mathbf{C}_{-}^{J}$ appears in the case of cubic tangency incoming from below (Figure $1(\mathrm{a})$ ) and map $\mathbf{C}_{+}^{J}$ arises in the case of cubic tangency incoming from above (Figure 1(b)). In the dissipative case $\sigma<1$, maps (1) with $|J| \ll 1$ were derived (using the rescaling method, [TY86]) in [GST96], where the corresponding formulas for the rescaled first return maps near homoclinic tangencies of arbitrary finite orders were obtained. It is worth mentioning that maps $\mathbf{C}_{+}^{J}$ and $\mathbf{C}_{-}^{J}$ were studied in GK88 for the dissipative case $|J|<1$, where bifurcation diagrams for fixed points and 2-periodic orbits were constructed analytically as well as strange attractors were analyzed numerically. Also maps (1) are good approximations of the corresponding rescaling first return maps $T_{k}$ when the return time $k$ is sufficiently large (in fact, $k$ is the period of a single-round periodic orbit under consideration). Then $(x, y)$ and $\left(M_{1}, M_{2}\right)$ are rescaled coordinates and parameters, respectively, which are defined in domains $\|(x, y)\|<D_{k}$ and $\left\|\left(M_{1}, M_{2}\right)\right\|<P_{k}$, where $D_{k}, P_{k} \rightarrow+\infty$ as $k \rightarrow \infty$, the Jacobian $J$ is proportional to $\sigma^{k}$ (i.e. $|J| \ll 1$ in the dissipative case and $J=1$ in the conservative case), and the error terms are of order $O\left(\lambda^{k}\right)$.

In the present paper we study bifurcations of single-round periodic orbits in the case of two-dimensional symplectic maps with cubic homoclinic tangencies. We put emphasis on bifurcations leading to the birth of elliptic periodic orbits. The main results can be briefly formulated as follows

Theorem 1. Let $f_{\mu}, \mu=\left(\mu_{1}, \mu_{2}\right)$, be a two parameter family of symplectic maps unfolding generally the cubic homoclinic tangency to the saddle fixed point $O$ with eigenvalues $\lambda$ and $\lambda^{-1}$, where $|\lambda|<1$. Let $V$ be a small neighborhood of the origin in the $\left(\mu_{1}, \mu_{2}\right)$-plane. Then the following holds

1. In $V$, there exists a bifurcation curve (semicubical parabola) $H_{0}$ with the cusp-point $\mu=(0,0)$ such that for $\mu \in H_{0} \backslash\{(0,0)\}$, map $f_{\mu}$ has a quadratic homoclinic tangency branched from the initial cubic tangency.

2. In $V$, there exists an infinite sequence of elliptic zones $E_{k}$ accumulated to $H_{0}$ as $k \rightarrow \infty$ such that for $\mu \in E_{k}$, map $f_{\mu}$ has an elliptic single-round orbit of period $k$. The boundary of $E_{k}$ consists of curves $L_{k}^{+}$and $L_{k}^{-}$ corresponding to the appearance of single-round periodic orbits with double eigenvalues +1 and -1 , respectively. 


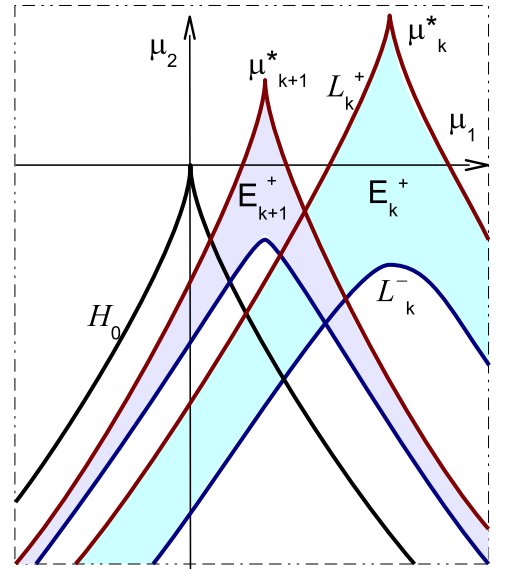

(a) «incoming from below» $\lambda>0$

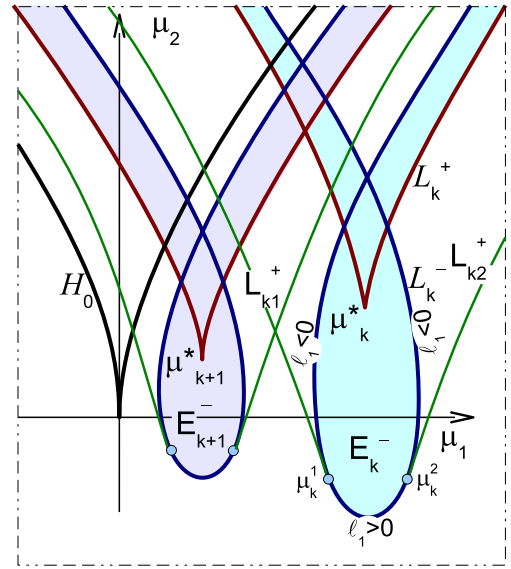

(b) «incoming from above» $\lambda>0$

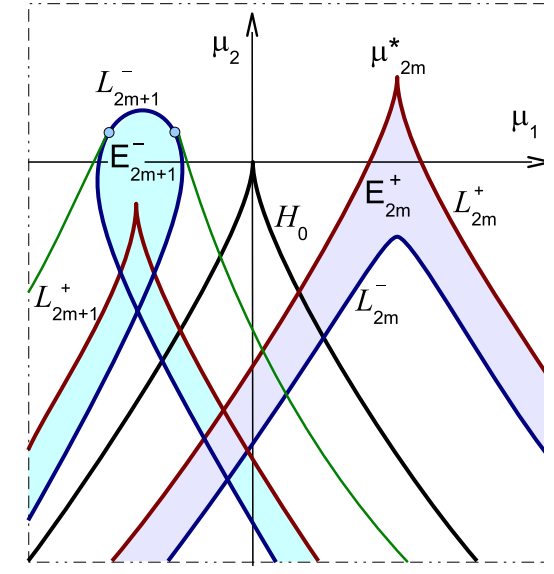

(c) $\lambda<0$

Figure 2: Main elements of the bifurcation diagram for single-round periodic orbits in the $\left(\mu_{1}, \mu_{2}\right)$-plane, where curve $H_{0}$ and elliptic zones $E_{k}^{+}$(a) and $E_{k}^{-}$(b) are shown. These zones alternate for $\lambda<0$ (c). Curves $L_{k}^{+}$correspond to nondegenerate conservative fold bifurcations except for cusp-points $\mu_{k}^{*}$ of conservative pitch-fork bifurcations. Curves $L_{k}^{-}$correspond to nondegenerate conservative period doubling bifurcations except for points $\mu_{k}^{1}$ and $\mu_{k}^{2}$ of curve $L_{k}^{-}$of zone $E_{k}^{-}$where the first Lyapunov value $\ell_{1}$ vanishes. Points $\mu_{k}^{1}$ and $\mu_{k}^{2}$ are the endpoints of bifurcation curves $L_{k 1}^{+}$and $L_{k 2}^{+}$that correspond to conservative fold bifurcations of double-round periodic orbits (2-periodic orbits in the first return maps).

The elliptic orbit is generic for all values of $\mu \in E_{k}$, except for those belonging to the strong 1:3 and 1:4 resonance curves $L_{k}^{2 \pi / 3}$ and $L_{k}^{\pi / 2}$ (the orbit has eigenvalues $e^{ \pm i 2 \pi / 3}$ and $e^{ \pm i \pi / 2}$ ) and the non-twist curve $L_{k}^{(0)}$ (the first Birkhoff coefficient equals zero).

An illustration to Theorem 1 is shown in Figure 2. We see that the bifurcation diagrams for single-round periodic orbits are different in the cases (a) $\lambda>0$ and the tangency is incoming from below; (b) $\lambda>0$ and the tangency is incoming from above; (c) $\lambda<0$. Correspondingly, we label zones $E_{k}$ as $E_{k}^{+}$(squids) in the case (a) and $E_{k}^{-}$ (cockroaches) in the case (b). Notice that in the case $\lambda<0$ zones $E_{k}$ of different types alternate depending on the parity of $k$, and, therefore, for more definiteness, we enumerate these zones as $E_{2 m}^{+}, E_{2 m+1}^{-}, \ldots$.

We prove Theorem 1 at the end of Section 2 (item 1) and Section 3 (item 2). The proof of item 2 of Theorem 1 is mainly based on the rescaling results of Lemma 4 which show that the corresponding first return map $T_{k}$ can be brought, by linear changes of coordinates and parameters, to a regular map asymptotically close as $k \rightarrow \infty$ to the conservative cubic Hénon map of the form (1) with $J=1$. Moreover, we obtain map $\mathbf{C}_{+}^{1}$ in the case of incoming from below tangency and map $\mathbf{C}_{-}^{1}$ in the case of incoming from above tangency (in the case $\lambda<0$, maps $\mathbf{C}_{+}^{1}$ and $\mathbf{C}_{-}^{1}$ appear, respectively, for even and odd numbers $k$ ). Bifurcations of fixed points and accompanied bifurcations of 2-periodic orbits for these conservative cubic Hénon maps are described in Section 3.1. The corresponding bifurcation diagrams are shown in Figures 5 and 6 for the maps $\mathbf{C}_{+}^{1}$ and $\mathbf{C}_{-}^{1}$, respectively. We also give the analytical expressions for the corresponding bifurcation curves, see formulas (16). After this, the proof of Theorem 1 becomes quite straightforward, see Section 3.3 .

Note that in the symplectic case, one of the main questions when studying elliptic points is related to their generic properties. In the case of the conservative Hénon map $\bar{x}=y, \bar{y}=M-x-y^{2}$, the problem of genericity (stability) of elliptic fixed points was completely solved in Bir87, see also SV09. Concerning the conservative cubic Hénon maps, the problem of genericity (stability) of fixed elliptic points has been (almost completely) solved in DM00, where conditions for the KAM-stability of these points were obtained. Note also that bifurcations of lower-periodic orbits (of period 1, 2, 3) were also studied in [DM00].

Concerning 4-periodic orbits, in Section 4 we carry out the corresponding bifurcation analysis paying main attention to those phenomena which are connected with the peculiarities of bifurcations of fixed points with eigenvalues $e^{ \pm i \pi / 2}$, i.e. 1:4 resonance. We perform this study separately for map $\mathbf{C}_{+}^{1}$ in Section 4.1 and for map $\mathbf{C}_{-}^{1}$ in Section 4.2 . 
Note that the main complex local normal form of a map having a fixed point with eigenvalues $e^{ \pm i \pi / 2}$ can be written as follows, Arn96,

$$
\bar{\zeta}=i(1+\beta) \zeta+i B_{1}|\zeta|^{2} \zeta+i B_{03} \zeta^{* 3}+O\left(|\zeta|^{5}\right),
$$

where $\beta$ is a parameter characterizing deviation of the angle argument $\varphi$ of eigenvalues of the fixed point from $\pi / 2$ $(\varphi=\beta+\pi / 2)$, the coefficients $B_{1}:=B_{1}(\beta)$ and $B_{03}:=B_{03}(\beta)$ are real and smoothly depend on $\beta$. Then the following conditions, Arn96,

$$
\left|B_{03}\right| \neq 0, \quad A:=\frac{\left|B_{1}(0)\right|}{\left|B_{03}(0)\right|} \neq 1
$$

imply that bifurcations of the fixed point in (2) are nondegenerate.

We show that conditions (3) can be violated for both maps $\mathbf{C}_{+}^{1}$ and $\mathbf{C}_{-}^{1}$. Namely, the case $B_{03}=0$ occurs for map $\mathbf{C}_{+}^{1}$ and the equality $A=1$ can hold for map $\mathbf{C}_{-}^{1}$. This means that the structure of $1: 4$ resonance is different in these maps and we study the accompanied bifurcation phenomena in the present paper.

Remark 1. As far as we know, the case $B_{03}=0$, when the fixed point in the normal form (2) is a nonlinear center (map (2) without $O\left(|z|^{5}\right)$ terms is a nonlinear rotation) was not considered before. Nevertheless, this case is very interesting from various points of view. In particular, the problem of the existence of quasi-central elliptic points in reversible analytical maps is very important. Here, bifurcations of such points (when the map is not conservative) lead to the birth of pairs of periodic orbits sink-source and, as a consequence, to the break-down of conservativity and appearance of the so-called mixed dynamics GST97, LS04, DGGLS13, even inside symmetric elliptic islands GLRT14.

Remark 2. The case $A=1$ of 1:4 resonance was considered in Bir87] for the conservative Hénon map, see also SV09]. However, for the cubic Hénon map $\mathbf{C}_{-}^{1}$, the case $A=1$ is of another (more complicated) structure. In particular, proper bifurcations of a fixed point with eigenvalues $e^{ \pm i \pi / 2}$ are replaced here by extrinsic bifurcations of some 4 -periodic orbits (thus, 1:4 resonance demonstrates certain non-twist properties).

In the present paper we only study the most interesting and principal peculiarities of 1:4 resonance in maps $\mathbf{C}_{+}^{1}$ and $\mathbf{C}_{-}^{1}$. In addition to the analytical considerations, we also display certain numerical results in Figures 9 and 11 . We plan to continue to study this in more detail in the nearest future.

The paper is organized as follows. In Section 2 we start with the statement of the problem and also present the results on the normal forms of symplectic saddle maps (Lemmas 1 and 2) and the normal form of global map $T_{1}$ defined near a homoclinic point (Lemma 3). We also prove item 1 of Theorem 1 in Section 2, see Proposition 1 In Section 3 we complete the proof of Theorem 14. The main technical result there is the Rescaling Lemma, Lemma 4. which shows that the first return maps can be written, in some rescaling coordinates, as maps asymptotically close to the conservative cubic Hénon maps $\mathbf{C}_{+}^{1}$ and $\mathbf{C}_{-}^{1}$. Once we know the structure of bifurcations of fixed points in these maps we can easily recover bifurcations of single-round periodic orbits and, hence, prove the theorem. In Section 4 we consider the problem on the structure of 1:4 resonance in maps $\mathbf{C}_{+}^{1}$ and $\mathbf{C}_{-}^{1}$.

\section{Statement of the problem.}

Let $f_{0}$ be a $C^{r}$-smooth, $r \geq 5$, two-dimensional symplectic diffeomorphism which satisfies the following conditions:

(A) $f_{0}$ has a saddle fixed point $O$ with eigenvalues $\lambda$ and $\lambda^{-1}$, where $|\lambda|<1$;

(B) the invariant manifolds $W^{u}(O)$ and $W^{s}(O)$ have a cubic homoclinic tangency at the points of some homoclinic orbit $\Gamma_{0}$.

We distinguish two types of cubic tangencies of $W^{u}(O)$ and $W^{s}(O)$ at a homoclinic point: the tangency of first type (incoming from above) and the tangency of the second type (incoming from below). Both these types are shown in Figure 1, When the eigenvalue $\lambda$ is positive the type of tangency remains the same for all points of the homoclinic orbit. However, if $\lambda$ is negative the incoming from above and incoming from below tangencies alternate from point to point.

Let $\mathcal{H}_{2}$ be a (codimension two) bifurcation surface composed of symplectic $C^{r}$-maps close to $f_{0}$ and such that every map of $\mathcal{H}_{2}$ has a close to $\Gamma_{0}$ homoclinic orbit at whose points the manifolds $W^{u}(O)$ and $W^{s}(O)$ have a cubic 

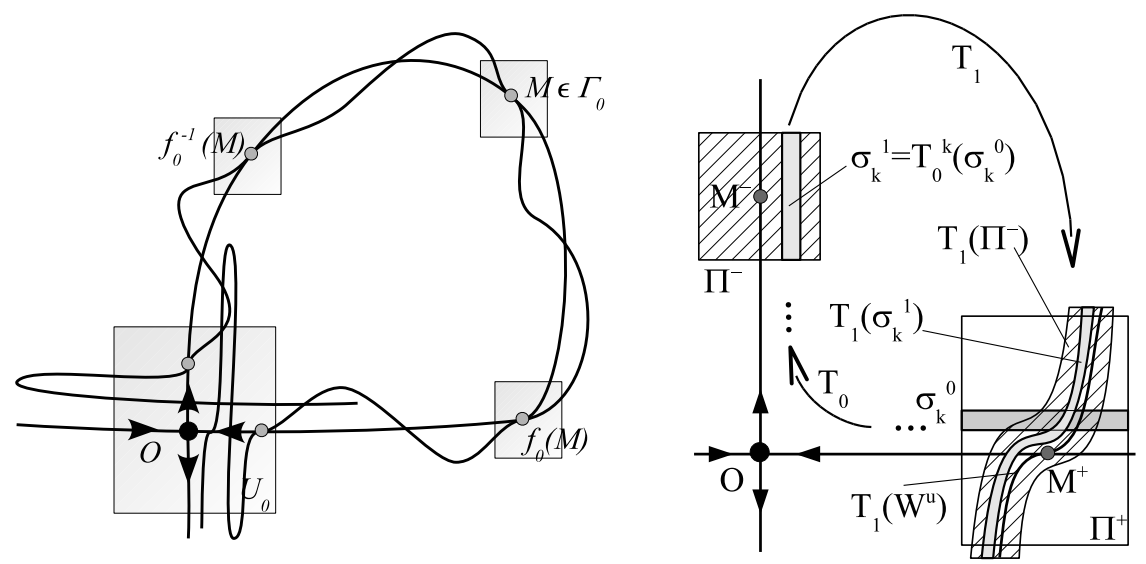

Figure 3: (a) An example of planar map having a cubic tangency at the points of a homoclinic orbit $\Gamma_{0}$. Some of these homoclinic points are shown as grey circles. Also a small neighborhood of the set $O \cup \Gamma_{0}$ is shown as the union of the squares; (b) construction of first return map $T_{k}=T_{1} T_{0}^{k}$.

tangency. Let $f_{\varepsilon}$ be a family of symplectic $C^{r}$-maps that contains map $f_{0}$ at $\varepsilon=0$. We suppose that the family depends smoothly on parameter $\varepsilon$ and satisfies the following condition:

(C) the family $f_{\varepsilon}$ is transverse to $\mathcal{H}_{2}$ at $\varepsilon=0$.

It is natural to assume that condition $\mathbf{C}$ holds for a two parameter family $f_{\varepsilon}$, where $\varepsilon=\left(\mu_{1}, \mu_{2}\right)$ and $\mu_{1}$ and $\mu_{2}$ are some parameters which split generally the initial cubic tangency of the manifolds $W^{u}(O)$ and $W^{s}(O)$ at some homoclinic point. We consider this question in more detail when proving Lemma 3 and we deduce that, without loss of generality, one can assume that $\varepsilon \in \mathbb{R}^{2}$.

Let $U$ be a sufficiently small fixed neighborhood of $O \cup \Gamma_{0}$. It consists of a small disk $U_{0}$ containing $O$ and a number of small disks $u_{i}, i=1,2, \ldots$, surrounding those points of $\Gamma_{0}$ that do not lie in $U_{0}$ (see Figure 3(a)). We call a periodic or homoclinic to $O$ orbit entirely lying in $U$ to be $p$-round if it has exactly $p$ intersection points with every disk $u_{i}$. For $p=1$ and $p=2$, we use the terms single-round and double-round orbits, respectively.

In the present paper we study bifurcations of single-round periodic orbits in the families $f_{\varepsilon}$ (we keep in mind that $\left.\varepsilon=\left(\mu_{1}, \mu_{2}\right)\right)$. Note that every point of such an orbit can be considered as a fixed point of the corresponding first return map. Such a map is usually constructed as a superposition $T_{k}=T_{1} T_{0}^{k}$ of two maps $T_{0} \equiv T_{0}(\varepsilon)$ and $T_{1} \equiv T_{1}(\varepsilon)$, see Figure 3(b). Map $T_{0}$ is called local map and it is the restriction of $f_{\varepsilon}$ onto $U_{0}$, i.e. $\left.T_{0}(\varepsilon) \equiv f_{\varepsilon}\right|_{U_{0}}$. Map $T_{1}$ is called global map, it is defined as $T_{1} \equiv f_{\varepsilon}^{q}$ with an integer $q$ and maps a small neighborhood $\Pi^{-} \subset U_{0}$ of some point $M^{-} \in W_{l o c}^{u}(O)$ of orbit $\Gamma_{0}$ into a neighborhood $\Pi^{+} \subset U_{0}$ of another point $M^{+} \in W_{\text {loc }}^{s}(O)$ of $\Gamma_{0}$ such that $f_{0}^{q}\left(M^{-}\right)=M^{+}$. Thus, any fixed point of $T_{k}$ is a point of a single-round periodic orbit of period $k+q$ for $f_{\varepsilon}$.

In order to study maps $T_{k}$ for all sufficiently large integer $k$, it is very important to have good coordinate representations for both maps $T_{0}$ and $T_{1}$, especially it concerns local map $T_{0}$ and its iterations $T_{0}^{k}$ for large $k$.

We use in $U_{0}$ the canonical coordinates $(x, y)$ given by the following lemma.

Lemma 1. GG09 For any given integer $n$ (such that $n<r / 2$ or $n$ is arbitrary for $r=\infty$ or $r=\omega$ (the real analytic case)), there is a canonical change of coordinates, of class $C^{r}$ for $n=1$ or $C^{r-2 n}$ for $n \geq 2$, that brings $T_{0}$ to the following form 1

$$
\begin{aligned}
& \bar{x}=\lambda x M_{n}(x y)+x|x y|^{n} O(|x|+|y|), \\
& \bar{y}=\lambda^{-1} y M_{n}^{-1}(x y)+y|x y|^{n} O(|x|+|y|),
\end{aligned}
$$

where $M_{n}(x y)=1+\beta_{1} \cdot x y+\cdots+\beta_{n} \cdot(x y)^{n}$ and $\beta_{i}$ are the Birkhoff coefficients.

\footnotetext{
${ }^{1}$ Note that the smoothness of these changes with respect to parameters can be $C^{r-2}$ for $n=1$ or $C^{r-2 n-2}$ for $n \geq 2$, respectively, see more details in GST07, SSTC98.
} 
The normal forms (44) are very suitable for effective calculations of maps $T_{0}^{k}:\left(x_{0}, y_{0}\right) \rightarrow\left(x_{k}, y_{k}\right)$ with sufficiently large integer $k$. Thus, the following result is valid.

Lemma 2. GG09 Let $T_{0}$ be given by (4), then map $T_{0}^{k}$ can be written, for any integer $k$, as follows

$$
\begin{aligned}
& x_{k}=\lambda^{k} x_{0} \cdot R_{n}^{(k)}\left(x_{0} y_{k}, \varepsilon\right)+\lambda^{n k} P_{n}^{(k)}\left(x_{0}, y_{k}, \varepsilon\right), \\
& y_{0}=\lambda^{k} y_{k} \cdot R_{n}^{(k)}\left(x_{0} y_{k}, \varepsilon\right)+\lambda^{n k} Q_{n}^{(k)}\left(x_{0}, y_{k}, \varepsilon\right),
\end{aligned}
$$

where $R_{n}^{(k)} \equiv 1+\tilde{\beta}_{1}(k) \lambda^{k} x_{0} y_{k}+\cdots+\tilde{\beta}_{n}(k) \lambda^{n k}\left(x_{0} y_{k}\right)^{n}, \tilde{\beta}_{i}(k), i=1, \ldots, n$, are some $i$-th degree polynomials of $k$ with coefficients depending on $\beta_{1}, \ldots, \beta_{i}$, and the functions $P_{n}^{(k)}, Q_{n}^{(k)}=o\left(x_{0}^{n} y_{k}^{n}\right)$ are uniformly bounded in $k$ along with all their derivatives by coordinates up to order either $(r-2)$ for $n=1$ or $(r-2 n-1)$ for $n \geq 2$.

Remark 3. The main case $n=1$ of Lemmas 1 and 2, related to the so-called main normal form of a saddle map, was proved in the papers GS00, GST07. Analogues of Lemmas 1 and 2 in the case of area-preserving and orientationreversing maps were proved in [DGG15].

Remark 4. Normal form (4) can be considered as a certain finitely smooth approximation of the following analytical Moser normal form

$$
\bar{x}=\lambda(\varepsilon) x \cdot M(x y, \varepsilon), \quad \bar{y}=\lambda^{-1}(\varepsilon) y \cdot M^{-1}(x y, \varepsilon),
$$

which takes place for $\lambda>0$ [Mos56], where $M(x y, \varepsilon)=1+\sum_{i=1}^{\infty} \beta_{i} \cdot(x y)^{i}$. Accordingly, relation (5) looks as a very good approximation for the corresponding formulas in the analytical case, GS97,

$$
x_{k}=\lambda^{k} x_{0} \cdot R^{(k)}\left(x_{0} y_{k}, \varepsilon\right), y_{0}=\lambda^{k} y_{k} \cdot R^{(k)}\left(x_{0} y_{k}, \varepsilon\right),
$$

where $R^{(k)} \equiv 1+\sum_{i=1}^{\infty} \tilde{\beta}_{i}(k) \lambda^{n k}\left(x_{0} y_{k}\right)^{i}$ and $\tilde{\beta}_{1}(k)=\beta_{1} k, \tilde{\beta}_{2}(k)=\beta_{2} k-\frac{1}{2} \beta_{1}^{2} k^{2}$, etc.

In the coordinates of Lemma 1] we have that $M^{+}=\left(x^{+}, 0\right), M^{-}=\left(0, y^{-}\right)$. Without loss of generality, we assume that $x^{+}>0$ and $y^{-}>0$. Let neighborhoods $\Pi^{+}$and $\Pi^{-}$of the homoclinic points $M^{+}$and $M^{-}$, respectively, be sufficiently small such that $T_{0}\left(\Pi^{+}\right) \cap \Pi^{+}=\emptyset$ and $T_{0}^{-1}\left(\Pi^{-}\right) \cap \Pi^{-}=\emptyset$. Then, as usually (see e.g. GS73), the successor map from $\Pi^{+}$into $\Pi^{-}$by orbits of $T_{0}$ is defined, for all sufficiently small $\varepsilon$, on the set consisting of infinitely many strips $\sigma_{k}^{0} \equiv \Pi^{+} \cap T_{0}^{-k} \Pi^{-}, k=\bar{k}, \bar{k}+1, \ldots$ The image of $\sigma_{k}^{0}$ under $T_{0}^{k}$ is the strip $\sigma_{k}^{1}=T_{0}^{k}\left(\sigma_{k}^{0}\right) \equiv \Pi^{-} \cap T_{0}^{k} \Pi^{+}$. As $k \rightarrow \infty$, the strips $\sigma_{k}^{0}$ and $\sigma_{k}^{1}$ accumulate to $W_{l o c}^{s}$ and $W_{l o c}^{u}$, respectively.

We can write global map $T_{1}(\varepsilon): \Pi^{-} \rightarrow \Pi^{+}$as follows (in the coordinates of Lemma 1)

$$
\bar{x}-x^{+}=F\left(x, y-y^{-}, \varepsilon\right), \quad \bar{y}=G\left(x, y-y^{-}, \varepsilon\right),
$$

where $F(0,0,0)=0, G(0,0,0)=0$ and

$$
\frac{\partial G(0)}{\partial y}=0, \quad \frac{\partial^{2} G(0)}{\partial y^{2}}=0, \quad \frac{\partial^{3} G(0)}{\partial y^{3}}=6 d \neq 0 .
$$

These relations follow from condition (B), which means that for $\varepsilon=0$ curve $T_{1}\left(W_{l o c}^{u}\right):\left\{\bar{x}-x^{+}=F\left(0, y-y^{-}, 0\right), \bar{y}=\right.$ $\left.G\left(0, y-y^{-}, 0\right)\right\}$ has a cubic tangency with $W_{l o c}^{s}:\{\bar{y}=0\}$ at $M^{+}$. When the parameters vary, this tangency can split and, by condition (C), family (7) unfolds generally the initial cubic tangency. In this case, global map $T_{1}$ can be written in a certain normal form that the following lemma shows.

Lemma 3. If condition $(\mathbf{C})$ holds, then map $T_{1}(\varepsilon)$ can be brought to the following form

$$
\begin{aligned}
& \bar{x}-x^{+}=a x+b\left(y-y^{-}-\right)+O\left(x^{2}+\left(y-y^{-}\right)^{2}\right), \\
& \bar{y}=\mu_{1}+\mu_{2}\left(y-y^{-}\right)+c x+d\left(y-y^{-}\right)^{3}+O\left(x^{2}+|x|\left|y-y^{-}\right|+\left(y-y^{-}\right)^{4}\right),
\end{aligned}
$$

where

$$
b(\varepsilon) c(\varepsilon) \equiv-1
$$

and the coefficients $a, b, c, d$ as well as $x^{+}, y^{-}$depend smoothly (with the same smoothness as for the initial map (7)) on new parameters $\varepsilon$ such that $\mu_{1} \equiv \varepsilon_{1}$ and $\mu_{2} \equiv \varepsilon_{2}$ (the other parameters are not essential). 


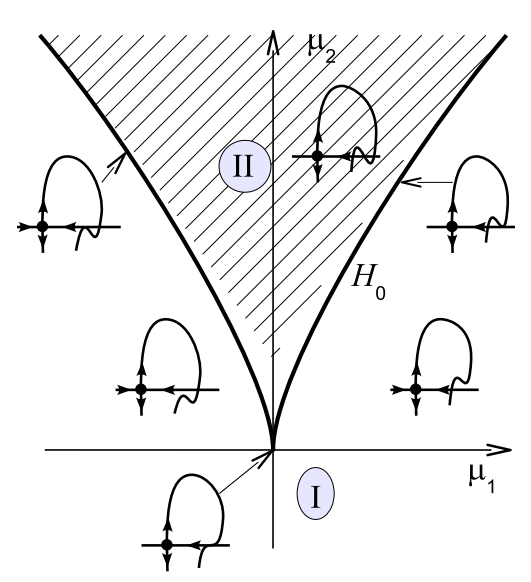

(a)

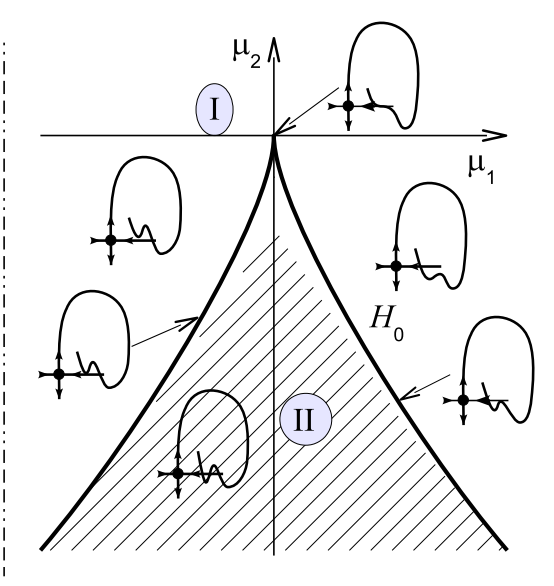

(b)

Figure 4: Curve $H_{0}$ in (a) incoming from above $(d<0)$ and (b) incoming from below $(d>0)$ cases. For $\mu \in I$, map $f_{\mu}$ has only one transverse homoclinic orbit close to $\Gamma_{0}$, whereas, for $\mu \in I I, f_{\mu}$ has exactly 3 transverse homoclinic orbits.

Proof. By virtue of (8), when $x$ and $\varepsilon$ are small enough, equation $\partial^{2} G\left(x, y-y^{-}, \varepsilon\right) / \partial y^{2}=0$ can be solved for $y-y^{-}$. The solution has the form $y-y^{-}(\varepsilon)=\varphi(x, \varepsilon)$, where $\varphi(0, \varepsilon) \equiv 0$. Then, we can write the following Taylor expansion for function $G$ near curve $y-y^{-}(\varepsilon)=\varphi(x, \varepsilon)$ :

$$
G \equiv G(x, 0, \varepsilon)+\frac{\partial G(x, 0, \varepsilon)}{\partial y}\left(y-y^{-}-\varphi\right)+\frac{\partial^{3} G(x, 0, \varepsilon)}{\partial y^{3}}\left(y-y^{-}-\varphi\right)^{3}+O\left(\left(y-y^{-}-\varphi\right)^{4}\right) .
$$

Since $\varphi \equiv \varphi(x, \varepsilon)=O(x)$, we obtain that

$$
G \equiv E_{1}(\varepsilon)+c x+E_{2}(\varepsilon)\left(y-y^{-}\right)+d\left(y-y^{-}\right)^{3}+O\left(x^{2}+|x|\left|y-y^{-}\right|+\left(y-y^{-}\right)^{4}\right),
$$

where $E_{i}(0)=0$. Hence, map $T_{1}(\varepsilon)$ can be written in the following form

$$
\begin{aligned}
& \bar{x}-x^{+}=a x+b\left(y-y^{-}\right)+O\left(x^{2}+\left(y-y^{-}\right)^{2}\right), \\
& \bar{y}=E_{1}(\varepsilon)+c x+E_{2}(\varepsilon)\left(y-y^{-}\right)+d\left(y-y^{-}\right)^{3}+O\left(x^{2}+|x|\left|y-y^{-}\right|+\left(y-y^{-}\right)^{4}\right) .
\end{aligned}
$$

Then the equation of the piece of $T_{1}\left(W_{l o c}^{u}\right)$ in $\Pi^{+}$has the following form

$$
\begin{aligned}
& \bar{x}-x^{+}=b\left(y-y^{-}\right)+O\left(\left(y-y^{-}\right)^{2}\right), \\
& \bar{y}=E_{1}(\varepsilon)+E_{2}(\varepsilon)\left(y-y^{-}\right)+d\left(y-y^{-}\right)^{3}+O\left(\left(y-y^{-}\right)^{4}\right) .
\end{aligned}
$$

Condition (C) implies that $E_{i}(0)=0, E_{i}^{\prime}(0) \neq 0$ and the coefficients $E_{1}(\varepsilon)$ and $E_{2}(\varepsilon)$ can take any values from the ball $\|\varepsilon\| \leq \delta_{0}$, where $\delta_{0}>0$ is a small constant. Thus, the system $\mu_{1}=E_{1}(\varepsilon), \mu_{2}=E_{2}(\varepsilon)$ has always a solution and we can consider $\mu_{1}$ and $\mu_{2}$ as new parameters.

Due to Lemma 3, we can consider directly two parameter families $f_{\mu_{1}, \mu_{2}}$ of symplectic maps. It is easy to see from (9) that $\mu_{1}$ and $\mu_{2}$ are the splitting parameters of the manifolds $W^{s}\left(O_{\varepsilon}\right)$ and $W^{u}\left(O_{\varepsilon}\right)$ with respect to the homoclinic point $M^{+}$. Indeed, by (10), curve $l_{u}=T_{1}\left(W_{l o c}^{u} \cap \Pi^{-}\right)$has the equation

$$
l_{u}: y=\mu_{1}+\frac{\mu_{2}}{b}\left(x-x^{+}\right)+\frac{d}{b^{3}}\left(x-x^{+}\right)^{3}+o\left(\left(x-x^{+}\right)^{3}\right) .
$$

Thus, family $f_{\mu_{1}, \mu_{2}}$ is a general two parameter unfolding of the initial cubic tangency which occurs for $\mu_{1}=\mu_{2}=0$. For any such unfolding, curves $W_{l o c}^{s}$ and $T_{1}\left(W_{l o c}^{u}\right)$ must have a quadratic tangency for certain values of $\mu_{1}$ and $\mu_{2}$. It is true that for our family the following result, which implies item 1 of Theorem 1 holds.

Proposition 1. In the $\left(\mu_{1}, \mu_{2}\right)$-parameter plane, there exists a bifurcation curve $H_{0}$ :

$$
\mu_{1}= \pm 2 d\left[-\frac{\mu_{2}}{3 d}\left(1+O\left(\sqrt{\left|\mu_{2}\right|}\right)\right)\right]^{3 / 2}
$$

(see Figure 4) such that for $\mu \in H_{0}$, map $f_{\mu}$ has a close to $\Gamma_{0}$ homoclinic orbit with a quadratic tangency of the manifolds $W^{u}\left(O_{\mu}\right)$ and $W^{s}\left(O_{\mu}\right)$. 
Proof. Consider curve $l_{u}$ given by equation (11). If the curve has a tangency with the line $y=0$, the following system has solutions

$$
\begin{aligned}
& \mu_{1}+\frac{\mu_{2}}{b}\left(x-x^{+}\right)+\frac{d}{b^{3}}\left(x-x^{+}\right)^{3}+o\left(\left(\bar{x}-x^{+}\right)^{3}\right)=0, \\
& \mu_{2}+\frac{3 d}{b^{2}}\left(x-x^{+}\right)^{2}+o\left(\left(\bar{x}-x^{+}\right)^{2}\right)=0 .
\end{aligned}
$$

We solve the second equation for $\left(x-x^{+}\right)$, put it into the first equation, and we obtain the equation of curve $H_{0}$. Note that the tangency is always quadratic except for the case $x-x^{+}=0$ which corresponds to a cubic tangency at $\mu_{1}=\mu_{2}=0$.

\section{On bifurcations of single-round periodic orbits}

The main goal in the present paper is to study bifurcations of single-round periodic orbits of family $f_{\mu_{1}, \mu_{2}}$. As said above, it is equivalent to the study of bifurcations of fixed points in the first return maps $T_{k}=T_{1} T_{0}^{k}$ for every sufficiently large integer $k\left(k=k_{0}, k_{0}+1, \ldots\right)$. We apply the rescaling method in order to find the rescaled normal forms for these maps in the following lemma.

Lemma 4. [Rescaling Lemma for cubic homoclinic tangencies]

For every sufficiently large $k$, the first return map $T_{k}$ can be brought, by a linear transformation of coordinates and parameters, to the following form

$$
\begin{aligned}
& \bar{x}=y+O\left(|\lambda|^{k / 2}\right), \\
& \bar{y}=M_{1}-x+M_{2} y+\nu y^{3}+O\left(|\lambda|^{k / 2}\right),
\end{aligned}
$$

where

$$
\begin{gathered}
\nu=\operatorname{sign}\left(d \lambda^{k}\right) \\
M_{1}=\sqrt{|d| \mid}|\lambda|^{-k / 2} \lambda^{-k}\left(\mu_{1}-\lambda^{k}\left(y^{-}-c x^{+}\right)+O\left(k \lambda^{2 k}\right)\right) \\
M_{2}=\lambda^{-k}\left(\mu_{2}+f_{11} \lambda^{k} x^{+}+O\left(k \lambda^{2 k}\right)\right)
\end{gathered}
$$

and $f_{11}=G_{x y}(0)$.

Proof. By Lemmas 2 and 3 , the first return map $T_{k}=T_{1} T_{0}^{k}$ can be written as follows

$$
\begin{aligned}
\bar{x}-x^{+}= & a \lambda^{k} x+b\left(y-y^{-}\right)+O\left(k \lambda^{2 k}|x|+|\lambda|^{k}|x|\left|y-y^{-}\right|+\left(y-y^{-}\right)^{2}\right) \\
\lambda^{k} \bar{y}\left(1+k \lambda^{k} O(\bar{x}, \bar{y})\right)= & \mu_{1}+\mu_{2}\left(y_{1}-y^{-}\right)+d\left(y_{1}-y^{-}\right)^{3}+c \lambda^{k} x \\
& +O\left(k \lambda^{2 k}|x|+|\lambda|^{k}|x|\left|y-y^{-}\right|\right)+O\left(\left(y-y^{-}\right)^{4}\right)
\end{aligned}
$$

We shift the coordinates, $\xi=x-x^{+}+\alpha_{k}^{1}, \eta=y-y^{-}+\alpha_{k}^{2}$, where $\alpha_{k}^{1}=-a \lambda^{k} x^{+}+O\left(k \lambda^{2 k}\right), \alpha_{k}^{2}=-\frac{f_{12}}{3 d} \lambda^{k} x^{+}+O\left(k \lambda^{2 k}\right)$ and $f_{12}=\frac{1}{2} G_{x y^{2}}(0)$. Then system (14) is rewritten as follows

$$
\begin{aligned}
\bar{\xi}= & a \lambda^{k} \xi+b \eta+O\left(k \lambda^{2 k}|\xi|+|\lambda|^{k}|\xi||\eta|+\eta^{2}\right) \\
\left(1+k \lambda^{k} O(\bar{\eta})\right) \bar{\eta}= & \lambda^{-k}\left(\mu_{1}-\lambda^{k}\left(y^{-}-c x^{+}\right)+\rho_{k}^{1}\right)+\lambda^{-k}\left(\mu_{2}+f_{11} \lambda^{k} x^{+}+\rho_{k}^{2}\right) \eta \\
& +d \lambda^{-k} \eta^{3}+c x+O\left(k \lambda^{k}|\xi|+|\xi||\eta|\right)+\lambda^{-k} O\left(\eta^{4}\right)
\end{aligned}
$$

where $\rho_{k}^{1,2}=O\left(k \lambda^{2 k}\right)$ are small coefficients. Here the first equation of (15) does not contain constant terms and the quadratic term in $\eta^{2}$ vanishes in the second equation.

Finally, by means of the coordinate rescaling

$$
\xi=b \frac{|\lambda|^{k / 2}}{\sqrt{|d|}} x, \quad \eta=\frac{|\lambda|^{k / 2}}{\sqrt{|d|}} y
$$

we bring map (15) to the required form (12). 


\subsection{The description of bifurcations of fixed points in the cubic Hénon maps}

By the Rescaling Lemma 4, the following conservative cubic Hénon maps $\mathbf{C}_{+}^{1}: \bar{x}=y, \bar{y}=M_{1}-x+M_{2} y+y^{3}$ and $\mathbf{C}_{-}^{1}: \bar{x}=y, \bar{y}=M_{1}-x+M_{2} y-y^{3}$ have to be considered as certain normal forms for the first return maps in the cases $d \lambda^{k}>0$ and $d \lambda^{k}<0$, respectively. Thus, if $\lambda>0$, map $\mathbf{C}_{+}^{1}$ corresponds to the cubic homoclinic tangency with $d>0$ (the incoming from below tangency, see Figure 1(b)), while map $\mathbf{C}_{-}^{1}$ is related to the cubic homoclinic tangency with $d<0$ (the incoming from above tangency, see Figure 1(a)). In Figures 5 and 6, the main elements of the bifurcation diagrams for these cubic maps are presented.

The bifurcation curves in Figures 5 and 6 are found analytically (see e.g. GK88, DM00]) and their equations are as follows, where $\nu=+1$ and $\nu=-1$ correspond to the maps $\mathbf{C}_{+}^{1}$ and $\mathbf{C}_{-}^{1}$, respectively:

$$
\begin{aligned}
& L^{+}: \quad M_{1}^{2}=\frac{4}{27 \nu}\left(2-M_{2}\right)^{3} ; \\
& L^{-}: \quad M_{1}^{2}=-\frac{4}{27 \nu}\left(2+M_{2}\right)\left(4-M_{2}\right)^{2} ; \\
& L_{2}^{+}: \quad M_{1}^{2}=-\frac{4}{27}\left(M_{2}+4\right)^{3}, \quad \text { if } \nu=+1 ; \\
& L_{2}^{+i}, i=1,2: \quad M_{1}=(-1)^{i} 2\left(\frac{M_{2}+4}{3}\right)^{3 / 2}, M_{2}>-\frac{4}{3}, \quad \text { if } \nu=-1 ; \\
& L_{2}^{-i}, i=1,2: \quad M_{1}^{2}=\frac{1}{216 \nu}\left[12+M_{2}+(-1)^{i} \sqrt{9 M_{2}^{2}+24 M_{2}}\right]^{2}\left[-5 M_{2}-12+(-1)^{i} \sqrt{9 M_{2}^{2}+24 M_{2}}\right]
\end{aligned}
$$

where if $\left(M_{1}, M_{2}\right) \in L^{+}$, then there exists a fixed point with double eigenvalue +1 ; if $\left(M_{1}, M_{2}\right) \in L^{-}$, there exists a fixed point with double eigenvalue -1 ; if $\left(M_{1}, M_{2}\right) \in L_{2}^{+}$, there exists a 2-periodic orbit with double eigenvalue +1 ; if $\left(M_{1}, M_{2}\right) \in L_{2}^{-}$, there exists a 2-periodic orbit with double eigenvalue -1 .

The main bifurcations related to fixed points are as follows.

Bifurcation scenario in map $\mathbf{C}_{+}^{1}$, see Figure 5. Bifurcation curves $L^{+}$and $L^{-}$from (16) with $\nu=+1$ divide the $\left(M_{1}, M_{2}\right)$-parameter plane into 3 regions symmetric with respect to the $M_{2}$-axis. When parameters $M_{1}$ and $M_{2}$ are in region $\mathbf{1}$, map $\mathbf{C}_{+}^{1}$ has a unique fixed point $p_{1}$ which is a saddle-plus (with eigenvalues $\lambda$ and $\lambda^{-1}$, where $0<\lambda<1$ ). The transition $\mathbf{1} \Rightarrow \mathbf{2}$ corresponds to the birth of two (saddle and elliptic) fixed points. At $\left(M_{1}, M_{2}\right)=b_{1}$, the fixed point $p_{1}$ is a non-hyperbolic saddle with double eigenvalue +1 , and then this point falls into three (two saddle and one elliptic) fixed points in domain $\mathbf{2}$ under a conservative pitch-fork bifurcation. The transition $\mathbf{2} \Rightarrow \mathbf{3}$ corresponds to a nondegenerate period doubling bifurcation of the elliptic fixed point. Thus, for region $\mathbf{3}$, map $\mathbf{C}_{+}^{1}$ has three saddle (two saddle-plus and one saddle-minus) fixed points and one elliptic 2-periodic orbit. Further primary bifurcations, when crossing curves $L_{2}^{+}$and $L_{2}^{-}$, are related to conservative fold and period doubling bifurcations of 2-periodic orbits, respectively, and we do not discuss them here (see more details in GK88, DM00]).

Bifurcation scenario in map $\mathbf{C}_{-}^{1}$, see Figure 6. Bifurcation curves (16) with $\nu=-1$ divide the $\left(M_{1}, M_{2}\right)$ parameter plane into 15 regions including the regions $\mathbf{1}, \mathbf{2}, \mathbf{4}, \mathbf{6}$ and $\mathbf{7}$ which are symmetric with respect to the $M_{2}$-axis, while the other regions are pairwise symmetric (e.g $\mathbf{3}$ and $\underline{\mathbf{3}}$ ). For $\left(M_{1}, M_{2}\right)$ belonging to region $\mathbf{1}$, map $\mathbf{C}_{-}^{1}$ has a unique fixed point $q_{1}$ which is a saddle-minus (with eigenvalues $\lambda$ and $\lambda^{-1}$, where $-1<\lambda<0$ ). The transition $\mathbf{1} \Rightarrow \mathbf{2}$ through the segment $\left(b_{1}, b_{1}^{\prime}\right)$ of curve $L^{-}$corresponds to a subcritical period doubling bifurcation with the point $q_{1}$ : the point becomes elliptic and a saddle 2-periodic orbit emerges in its neighborhood. The transition $\mathbf{1} \Rightarrow \mathbf{3}$ (and symmetrically $\mathbf{1} \Rightarrow \underline{\mathbf{3}}$ ) corresponds to a nondegenerate conservative fold bifurcation under which saddle and elliptic 2-periodic orbits are born. The transition $\mathbf{3} \Rightarrow \mathbf{2}$ (as well as $\underline{\mathbf{3}} \Rightarrow \mathbf{2}$ ) corresponds to a supercritical period doubling bifurcation under which the elliptic 2-periodic orbit merges with the saddle fixed point and becomes an elliptic fixed point. Thus, in domain 2, map $\mathbf{C}_{-}^{1}$ has an elliptic fixed point and a saddle 2-periodic orbit. The transitions $\mathbf{3} \rightarrow \mathbf{3}^{\prime}, \mathbf{5} \rightarrow \mathbf{5}^{\prime}$ and $\mathbf{6} \rightarrow \mathbf{6}^{\prime}$ (and, symmetrically, $\underline{\mathbf{3}} \rightarrow \underline{\mathbf{3}^{\prime}}, \underline{\mathbf{5}} \rightarrow \underline{\mathbf{5}^{\prime}}$ and $\mathbf{6} \rightarrow \underline{\mathbf{6}^{\prime}}$ ) correspond to a period doubling of the elliptic 2-periodic orbit (it becomes a saddle 2-periodic orbit and an elliptic 4-periodic orbit is born in its neighborhood). We also illustrate bifurcations occurring at a passage around the point $b_{1}$ in Figure 7 (and, symmetrically, for the point $b_{1}^{\prime}$ ). Transitions through curve $L^{+}$at increasing $M_{2}$ correspond to the appearance of two new (saddle and elliptic) fixed points under 

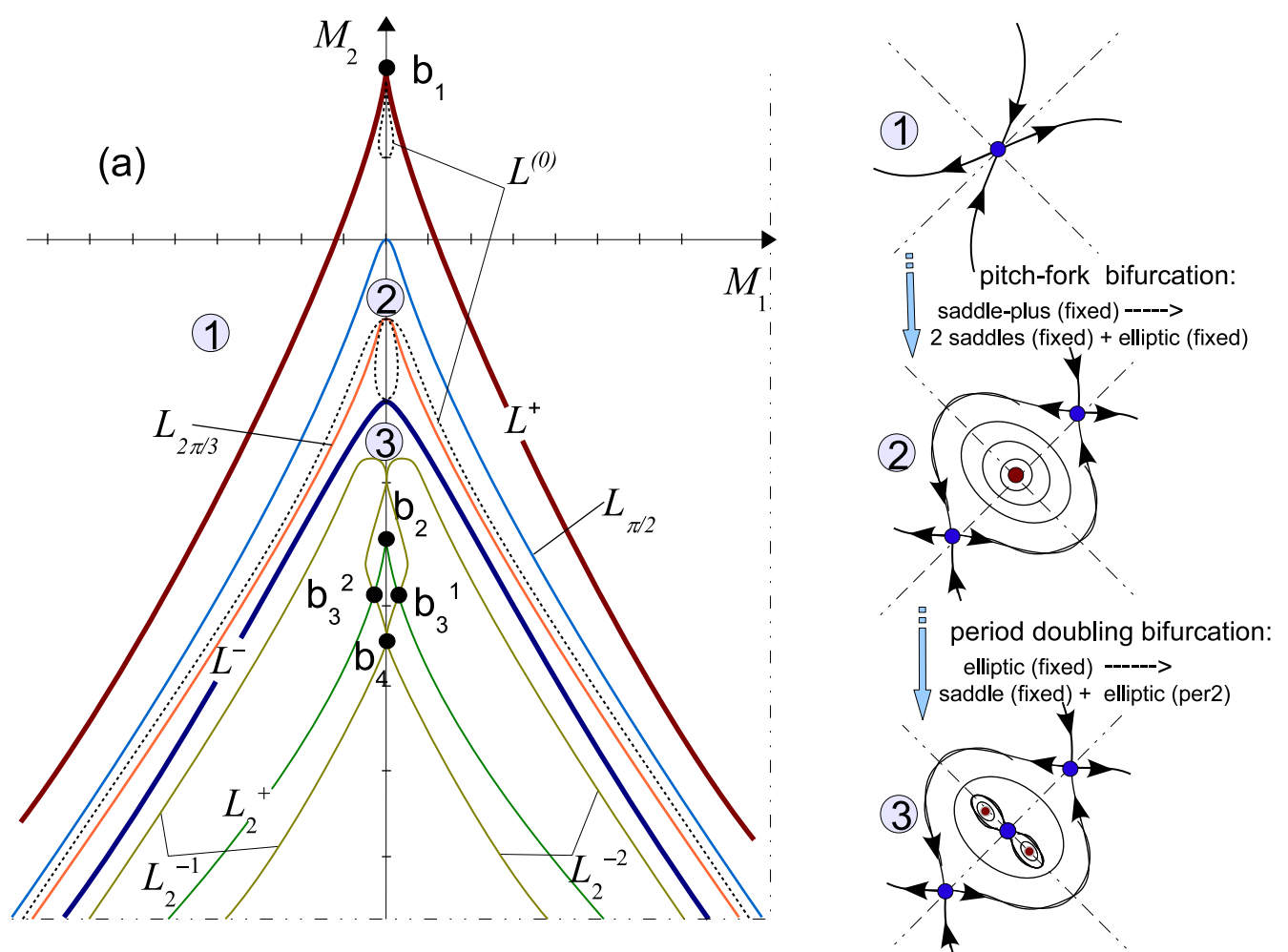

(b) Main symmetric bifurcations $\left(\mathrm{M}_{1}=0\right)$
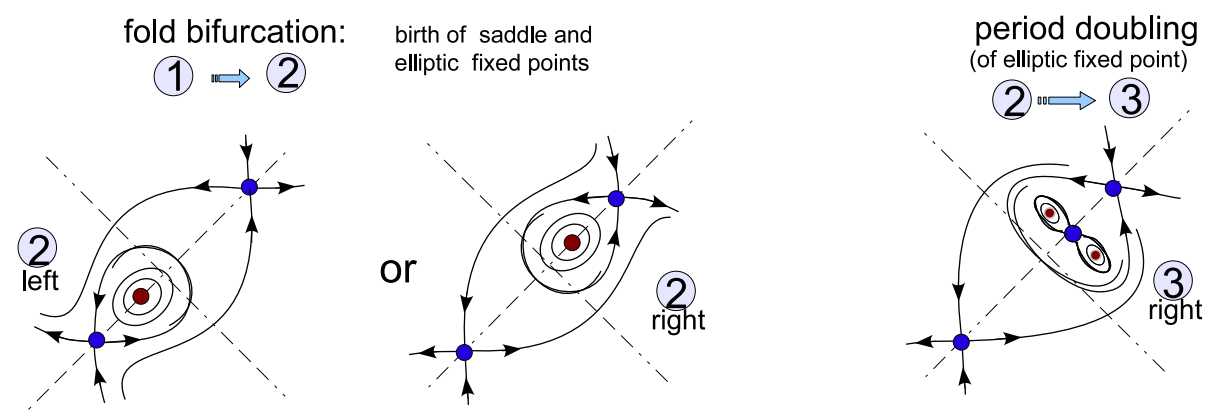

(c) some non-symmetric bifurcations

Figure 5: (a) The main elements of the bifurcation diagram for map $\mathbf{C}_{+}^{1}$. The equations of bifurcation curves $L^{+}, L^{-}$ are given by (16) with $\nu=1$. The equation of $L^{(0)}$ is given by (20) with "+". The codimension 2 bifurcation points $b_{i}$ are as follows: $b_{1}$ - a nonhyperbolic saddle fixed point with double eigenvalue +1 exists; $b_{2}$ and $b_{4}$ - two 2-periodic cycles with double eigenvalue -1 coexist; $b_{3}^{1,2}$ - two 2-periodic cycles, one with double eigenvalues -1 and the other with double eigenvalues +1 , coexist. Examples of $(\mathrm{b})$ symmetric $\left(M_{1}=0\right)$ and $(\mathrm{c})$ asymmetric $\left(M_{1} \neq 0\right)$ bifurcations are also shown. 

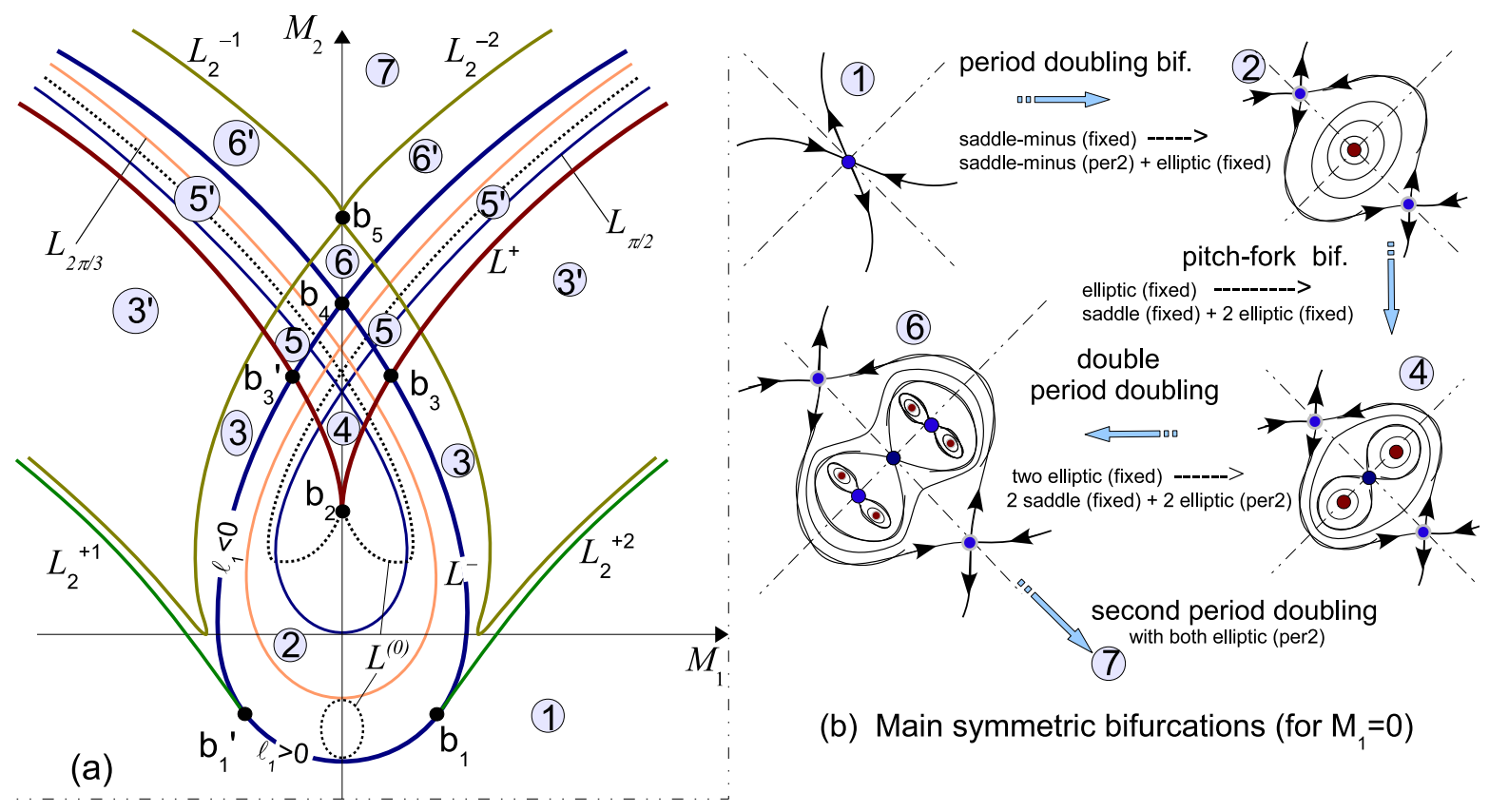

(b) Main symmetric bifurcations (for $M_{1}=0$ )
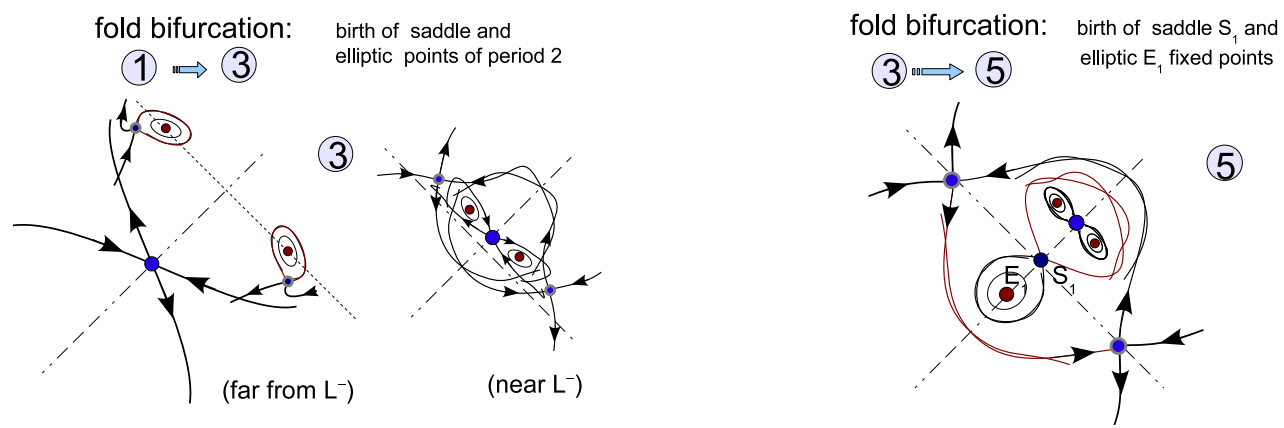

(c) some non-symmetric fold bifurcations

Figure 6: (a) The main elements of the bifurcation diagram for map $\mathbf{C}_{-}^{1}$. The equations of bifurcation curves $L^{+}$ and $L^{-}$are given by (16) with $\nu=-1$. The equation of $L^{(0)}$ is given by (20) with "-". The codimension 2 bifurcation points $b_{i}$ are as follows: $b_{1}$ and $b_{1}^{\prime}$ - there exists a fixed point with eigenvalues $(-1 ;-1)$ whose first Lyapunov value is zero but second is nonzero, these points divide curve $L^{-}$into the segments where the corresponding period doubling bifurcation is either subcritical (when crossing the piece $\left(b_{1}, b_{1}^{\prime}\right)$ of $L^{-}$) or supercritical (crossing outside $\left.\left(b_{1}, b_{1}^{\prime}\right)\right) ; b_{2}-$ a triple fixed point of elliptic type exists; $b_{3}$ and $b_{3}^{\prime}$ - two fixed points with eigenvalues $(-1,-1)$ and $(+1,+1)$ coexist; $b_{4}$ - two fixed points with eigenvalues $(-1,-1)$ coexist; $b_{5}$ - two 2 -periodic orbits with eigenvalues $(-1,-1)$ coexist. 


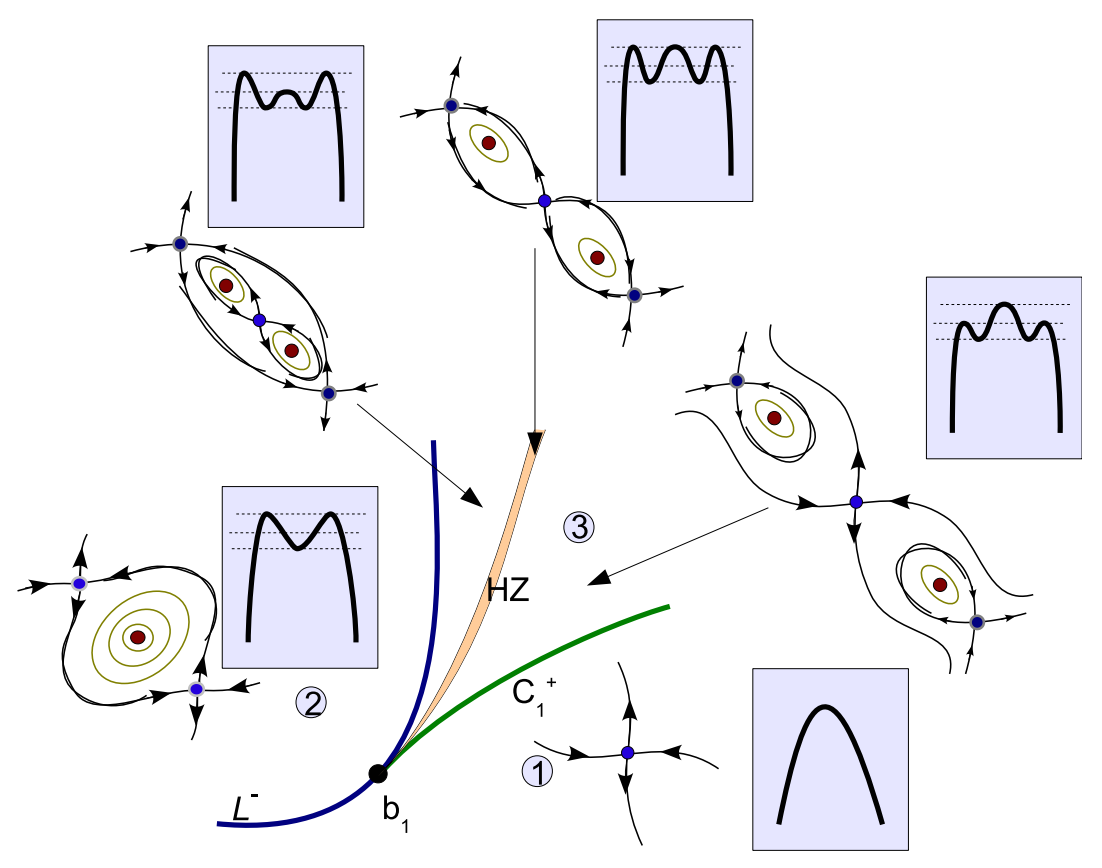

Figure 7: Bifurcations near point $b_{1}$ of the bifurcation diagram in Figure 6] Region $H Z$ (homo/heteroclinic zone) corresponds to the values of $\left(M_{1}, M_{2}\right)$ where the invariant manifolds of all saddles intersect. In a rough approximation, these bifurcations are similar to those of a two-dimensional Hamiltonian system whose potential function is symmetric and changes as shown.

a conservative fold bifurcation. Thus, in region $\mathbf{4}$, map $\mathbf{C}_{-}^{1}$ has three fixed points, two elliptic and one saddle. The elliptic fixed points undergo a period doubling bifurcation at transitions $\mathbf{4} \Rightarrow \underline{\mathbf{5}}$ and $\mathbf{5}^{\prime} \Rightarrow \mathbf{6}^{\prime}$ - for one of the points, or $\mathbf{4} \Rightarrow \mathbf{5}$ and $\underline{\mathbf{5}^{\prime}} \Rightarrow \underline{\mathbf{6}^{\prime}}$ - for the other point. We note that for $\left(M_{1}, M_{2}\right)=b_{2}$, there exists a triple (of elliptic type) fixed point which splits into three (two elliptic and one saddle) fixed points in region 4.

\subsection{On genericity of elliptic fixed points for the conservative cubic Hénon maps.}

In the $\left(M_{1}, M_{2}\right)$-parameter plane, bifurcation curves $L^{+}$and $L^{-}$bound those open regions of parameter values where elliptic fixed points of maps $\mathbf{C}_{+}^{1}$ and $\mathbf{C}_{-}^{1}$ exist. Such a fixed point is called generic if, first, $\varphi \neq 0, \pi, \pi / 2,2 \pi / 3$, i.e. no strong resonances occurs, and, second, in the main complex normal form

$$
\bar{z}=e^{i \varphi} z+i B_{1} z|z|^{2}+O\left(|z|^{4}\right)
$$

the first Birkhoff coefficient $B_{1}$ is nonzero. Note that if an elliptic point is generic, then it is stable. However, nongeneric elliptic points can be both stable (of elliptic type) and unstable (of saddle type, for example, a saddle with 6 or 8 separatrices if $\varphi=2 \pi / 3$ or $\varphi=\pi / 2)$.

The following curve

$$
L_{\varphi}: M_{1}^{2}= \pm \frac{4}{27}\left(M_{2}+\cos \varphi-3\right)^{2}\left(2 \cos \varphi-M_{2}\right)
$$

in the $\left(M_{1}, M_{2}\right)$-parameter plane defines those parameter values where map $\mathbf{C}_{ \pm}^{1}$ has a fixed point $E_{\varphi}$ with eigenvalues $e^{ \pm i \varphi}$ with $0<\varphi<\pi$. Accordingly, curves $L_{2 \pi / 3}$ and $L_{\pi / 2}$ of strong 1:3 and 1:4 resonances are resulted from (18) for $\cos \varphi=0$ and $\cos \varphi=-1 / 2$, respectively.

To find the conditions when the point $E_{\varphi}$ is non-generic, i.e. $B_{1}\left(E_{\varphi}\right)=0$, we use the formula for $B_{1}$ from [RT99], 
see formula (35) there. Then we obtain that $B_{1}=0$ for

$$
M_{2}=\frac{6 \cos ^{2} \varphi+3 \cos \varphi+1}{1+4 \cos \varphi} .
$$

This formula is true for both maps $\mathbf{C}_{+}^{1}$ and $\mathbf{C}_{-}^{1}$.

Equations (18) and (19) define in the $\left(M_{1}, M_{2}\right)$-parameter plane the so-called non-twist curve $L^{(0)}$ such that for $\left(M_{1}, M_{2}\right) \in L^{(0)}$, map $\mathbf{C}_{ \pm}^{1}$ has a degenerate elliptic fixed point (with $B_{1}=0$ ). These curves are shown in Figures 5 and 6. For both maps, the curves consist of two connected pieces, the infinite branches of $L^{(0)}$ asymptotically tend to the curve $L_{\varphi}$ with $\varphi=\arccos (-1 / 4)$. In the case of map $\mathbf{C}_{-}^{1}$, one of the branches of the curve $L^{(0)}$ has a selfintersection point $\left(M_{1}=0 ; M_{2}=3.2\right)$, where map $\mathbf{C}_{-}^{1}$ has simultaneously two degenerate elliptic fixed points (both with $\cos \varphi=-1 / 5)$. For map $\mathbf{C}_{+}^{1}$, there also exists a self-intersection point $\left(M_{1}=0, M_{2}=-1\right)$ of curve $L^{(0)}$. This point corresponds to the existence of a fixed point with eigenvalues $e^{ \pm i 2 \pi / 3}$ (1:3 resonance), which is KAM-stable in this case, [DM00]. Note that, in the case of map $\mathbf{C}_{-}^{1}$, the fixed point $O(0,0)$ at $M_{1}=0, M_{2}=-1$ has eigenvalues $e^{ \pm i 2 \pi / 3}$ and is also KAM-stable (here $L^{(0)}$ and $L_{2 \pi / 3}^{-}$are tangent). Note that the fixed point $E_{\varphi}$ is always nondegenerate (generic) if $-1 / 4 \cos \varphi<1 / 6$ in the case of map $\mathbf{C}_{+}^{1}$ and if $-1 / 2<\cos \varphi<-1 / 4$ in the case of map $\mathbf{C}_{-}^{1}$. We also note that curves $L^{(0)}$ can be written in the explicit form DM00] as follows

$$
729 M_{1}^{4} \pm\left(8 M_{2}^{3}-108 M_{2}^{2}-63 M_{2}+837\right) M_{1}^{2}-\frac{16}{27}\left(M_{2}+1\right)\left(5 M_{2}-16\right)^{2}\left(M_{2}-2\right)^{3}=0 .
$$

\subsection{End of proof of Theorem 1}

Now we can complete the proof of Theorem 1. Indeed, we only need to translate the results on the bifurcation diagrams obtained in the $\left(M_{1}, M_{2}\right)$-plane for the rescaling maps $\mathbf{C}_{+}^{1}$ and $\mathbf{C}_{-}^{1}$ into the initial $\left(\mu_{1}, \mu_{2}\right)$-parameter plane. We can easily make this using relations (13) between the initial and rescaling parameters. Then, using equations (16) for curves $L^{+}, L^{-}$and $L_{2}^{+}$, we obtain the following equations for the curves in the $\left(\mu_{1}, \mu_{2}\right)$-plane, where $\nu=\operatorname{sign}\left(d \lambda^{k}\right)$,

$$
\begin{aligned}
& L_{k}^{+}: \quad \mu_{1}=\lambda^{k}\left(y^{-}-c x^{+}+\ldots\right) \pm \frac{2}{\sqrt{|d|}}\left(\frac{\left(2-f_{11} x^{+}\right) \lambda^{k}-\mu_{2}}{3 \nu}\right)^{3 / 2}(1+\ldots) ; \\
& L_{k}^{-}: \quad \mu_{1}=\lambda^{k}\left(y^{-}-c x^{+}+\ldots\right) \pm \frac{2}{3 \sqrt{|d|}}\left(\frac{-\left(2+f_{11} x^{+}\right) \lambda^{k}-\mu_{2}}{3 \nu}\right)^{1 / 2}\left(\left(4-f_{11} x^{+}\right) \lambda^{k}-\mu_{2}\right)(1+\ldots) ; \\
& L_{2 k}^{+}: \quad \mu_{1}=\lambda^{k}\left(y^{-}-c x^{+} \ldots\right) \pm \frac{2}{\sqrt{|d|}}\left(\frac{-\left(4+f_{11} x_{1}^{+}\right) \lambda^{k}-\mu_{2}}{3}\right)^{3 / 2}(1+\ldots), \text { if } \nu=+1 ; \\
& L_{2 k}^{+i}:\left\{\begin{array}{l}
\mu_{1}=\lambda^{k}\left(y^{-}-c x^{+} \ldots\right)+(-1)^{i} \frac{2}{\sqrt{|d|}}\left(\frac{\left(4+f_{11} x^{+}\right) \lambda^{k}+\mu_{2}}{3}\right)^{3 / 2}(1+\ldots), \\
\text { where } \mu_{2}+f_{11} x^{+} \lambda^{k} \geq-\frac{4}{3} \lambda^{k} \text { and } i=1,2, \text { if } \nu=-1 .
\end{array}\right.
\end{aligned}
$$

Analogous formulas can be obtained for the other bifurcation curves, given in (16), (18) for $\varphi=\pi / 2,2 \pi / 3$, and (20). This completes the proof of Theorem 1

\section{On 1:4 resonance in the cubic Hénon maps}

The structure of the strong 1:3 resonance, i.e. the structure of bifurcations related to the existence of fixed points with eigenvalues $e^{ \pm i 2 \pi / 3}$, in the case of cubic maps $\mathbf{C}_{+}^{1}$ and $\mathbf{C}_{-}^{1}$ was studied in [DM00]. In this section we analyze bifurcations related to the existence of fixed points with eigenvalues $e^{ \pm i \pi / 2}$ (the main 1:4 resonance) in maps $\mathbf{C}_{+}^{1}$ and $\mathbf{C}_{-}^{1}$. We find conditions of nondegeneracy of the corresponding resonances and give a description of accompanying bifurcations including the main bifurcations in the degenerate cases.

Among the latter bifurcations we pay main attention to bifurcations of symmetric 4-periodic points, i.e. such 4-periodic orbits which has two points on the symmetry line $R:\{x=y\}$. We will call such orbits $R$-symmetric. 
Note that both maps $\mathbf{C}_{+}^{1}$ and $\mathbf{C}_{-}^{1}$ are reversible with respect to the involution $\mathcal{L}:\{x \rightarrow y, y \rightarrow x\}$, i.e. the maps preserve the form if one makes the coordinate change $\mathcal{L}$ and consider the inverse map. The line $R$ is the line of fixed points of involution $\mathcal{L}$ and, thus, $R$-symmetric periodic orbits can undergo nondegenerate bifurcations of two types: symmetry preserving parabolic (hold) bifurcations and symmetry breaking pitch-fork bifurcations. In the conservative case, there are two types of pitch-fork bifurcations: (i) when a symmetric elliptic fixed (periodic) point becomes a symmetric saddle fixed point and a pair of elliptic fixed points (symmetric each other with respect to $R$ ) is born, and (ii) when a symmetric saddle fixed (periodic) point becomes a symmetric elliptic fixed point and a pair of symmetric each other saddle fixed points is born.

We note that, in the case under consideration, there are nonsymmetric 4-periodic orbits whose structure and bifurcations can be important to understand some thin details of 1:4 resonance. In this case, we study these orbits only numerically.

\subsection{1:4 resonance in map $\mathrm{C}_{+}^{1}$.}

The map $\mathbf{C}_{+}^{1}$ has a unique fixed point with eigenvalues $e^{ \pm i \pi / 2}$ at values of parameters $M_{1}$ and $M_{2}$ belonging to the curve

$$
L_{\pi / 2}^{+}: M_{1}^{2}=-\frac{4}{27} M_{2}\left(M_{2}-3\right)^{2} .
$$

Its equation is obtained from (18) with the sign " + " and $\cos \varphi=0$.

It was found in Gon05] that the complex local normal form (2) near such a point has the following coefficients

$$
8 B_{1}(0)=3-3 M_{2}, \quad 8 B_{03}(0)=1+3 M_{2}
$$

and, thus, in (3)

$$
A=\frac{\left|3-3 M_{2}\right|}{\left|1+3 M_{2}\right|} .
$$

Since $M_{2} \leq 0$ in the case of curve $L_{\pi / 2}^{+}$, we always have $A>1$. Thus, in (3) only condition $\left|B_{03}(0)\right| \neq 0$ is violated at $M_{2}=-1 / 3$.

The main local bifurcations which occur here at transition of the parameters through curve $L_{\pi / 2}^{+}$(except for the two points with $\left.M_{2}=-1 / 3\right)$ are illustrated in Figure 8 Curve $L_{\pi / 2}^{+}$divides the $\left(M_{1}, M_{2}\right)$-parameter plane into two regions I and II. In both regions, the fixed point is generic elliptic, however, it is not generic for $\left(M_{1}, M_{2}\right) \in L_{\pi / 2}^{+}$ although the point is of elliptic type. The transition from I into II is accompanied by the appearance of a resonant 1:4 chain containing the saddle and elliptic 4-periodic cycles around the fixed point.

We note that in curve $L_{\pi / 2}^{+}$there are two points $p_{4}^{l}$ and $p_{4}^{r}$ with $M_{2}=-1 / 3$, where $B_{03}=0$. Now we consider in more detail a neighborhood of the point $p_{4}^{r}$ (point $p_{4}^{l}$ can be studied in the same way). We find that $p_{4}^{r}$ is an endpoint of two bifurcation curves $B_{4}^{r}$ and $\tilde{B}_{4}^{r}$. These two curves divide domain II into 3 domains, $\mathrm{II}_{a}, \mathrm{II}_{b}$ and $\mathrm{II}_{c}$. In domain $\mathrm{II}_{a}$, the 1:4 chain looks as in Figure 9 for $M_{1}=0.7, M_{2}=-0.5$. Thus, the chain contains the $R$-symmetric elliptic 4-periodic orbit (whose two points belong to the bisecting line $x=y$ ). At crossing the line $B_{4}^{r}$ (from $\mathrm{II}_{a}$ to $\mathrm{II}_{b}$ ), the elliptic orbit undergoes a pitch-fork bifurcation: it becomes a symmetric saddle 4-periodic orbit and a pair of symmetric each other elliptic 4-periodic orbits emerges. When the parameters change in zone $\mathrm{II}_{b}$, some bifurcations connected with the reconstruction of homo/heteroclinic structures take place, and, finally, the saddle 4-periodic orbit of the chain which is not $R$-symmetric undergoes a pitch-fork bifurcation at crossing curve $\tilde{B}_{4}^{r}$ : two elliptic 4-periodic orbits merge into it and the orbit becomes elliptic. Thus, in domain $\mathrm{II}_{c}$, the 1:4 chain looks as in Figure 9 for $M_{1}=0.75, M_{2}=-0.5$.

In Figure 10 we interpret these bifurcations by means of the following flow normal form (which is invariant under rotation by angle $\pi / 2$ )

$$
\dot{\zeta}=-4 i\left(-\beta \zeta+B_{1} \zeta|\zeta|^{2}+\mu \zeta^{* 3}+\hat{A} \zeta^{5}+B_{2}|\zeta|^{4} \zeta+\hat{C}|\zeta|^{2} \zeta^{* 3}+O\left(|\zeta|^{7}\right)\right),
$$

where $\beta$ and $\mu$ are small real parameters, coefficients $B_{1}, B_{2}, \hat{A}$ and $\hat{C}$ are real and $5 \hat{A} \equiv \hat{C}$ (in this case the divergence of (22) is zero, [Bir87]). Note that system (22) is also reversible, it is invariant under the time reversal $t \rightarrow-t$ and the following involutions: $\mathcal{R}_{1}:\{x \rightarrow y, y \rightarrow x\}, \mathcal{R}_{2}:\{x \rightarrow-y, y \rightarrow-x\}, \mathcal{R}_{3}:\{x \rightarrow-x, y \rightarrow y\}$ and 

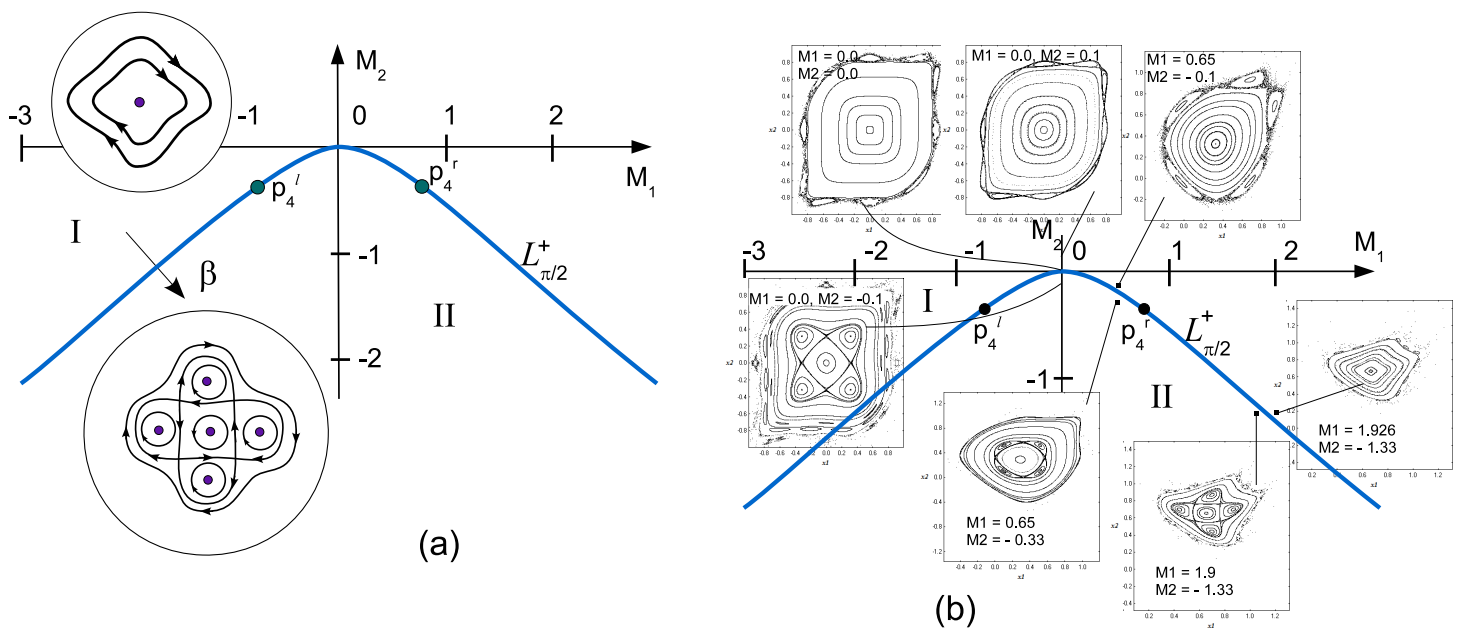

Figure 8: Local bifurcations at transition of the parameters through curve $L_{\pi / 2}^{+}$for (a) the flow normal form (2); (b) for map $\mathbf{C}_{+}^{1}$, the phase portraits are obtained numerically.

$\mathcal{R}_{4}:\{x \rightarrow x, y \rightarrow-y\}$. Accordingly, if $B_{1} \beta<0$, the system has 8 nontrivial equilibria lying in pairs in the 4 lines $\mathcal{R}_{1}, \ldots, \mathcal{R}_{4}$ of fixed points of the corresponding involutions.

We see that the theoretical results, Figure 10, are in good accordance with the numerical evidence for a parameter path $M_{2}=-0.5$ in Figure 9. Note that in Figure 10 the case (c) approximates the homo/heteroclinic zone of domain $\mathrm{II}_{b}$, where the invariant manifolds of the saddles are reconstructed from the (b)-configuration to the (d)configuration. Simultaneously when the parameters change in domain $\mathrm{II}_{b}$, we observe the effect of thinning of the 1:4 resonance chain. This is connected with the fact that the normal form (22) with $\mu=0$ and $C=0$ corresponds to a nonlinear center.

\section{$4.2 \quad 1: 4$ resonance in map $\mathrm{C}_{-}^{1}$}

The cubic map $\mathbf{C}_{-}^{1}$ has a fixed point with eigenvalues $e^{ \pm i \pi / 2}$ for $\left(M_{1}, M_{2}\right) \in L_{\pi / 2}^{-}$, where curve $L_{\pi / 2}^{-}$has equation

$$
L_{\pi / 2}^{-}: M_{1}^{2}=\frac{4}{27} M_{2}\left(M_{2}-3\right)^{2} .
$$

This equation is obtained from (18) with the sign "-" and $\cos \varphi=0$.

Note that curve $L_{\pi / 2}^{-}$has a self-intersection point $\left(M_{1}=0, M_{2}=3\right)$, and only in this moment the map has simultaneously two fixed points with eigenvalues $e^{ \pm i \pi / 2}$. The coefficients of the local complex normal form (2) are, Gon05,

$$
8 B_{1}=-3+3 M_{2}, 8 B_{03}=-1-3 M_{2} .
$$

Since $M_{2} \geq 0$ in curve $L_{\pi / 2}^{-}$, in (3) we have $B_{03}<0$ and

$$
A=\frac{\left|3-3 M_{2}\right|}{\left|1+3 M_{2}\right|} .
$$

Thus, both cases $A>1$ (when $0 \leq M_{2}<1 / 3$ ) and $A<1$ (when $M_{2}>1 / 3$ ) are possible here. In $L_{\pi / 2}^{-}$, there are two points $P^{+}\left(M_{1}=16 / 27, M_{2}=1 / 3\right)$ and $P^{-}\left(M_{1}=-16 / 27, M_{2}=1 / 3\right)$ where the case $A=1$ takes place. Note that if $A<1$, the fixed point $O$ is a saddle with 8 separatrices for $\left(M_{1}, M_{2}\right) \in L_{\pi / 2}^{-}$, and the main bifurcations are 


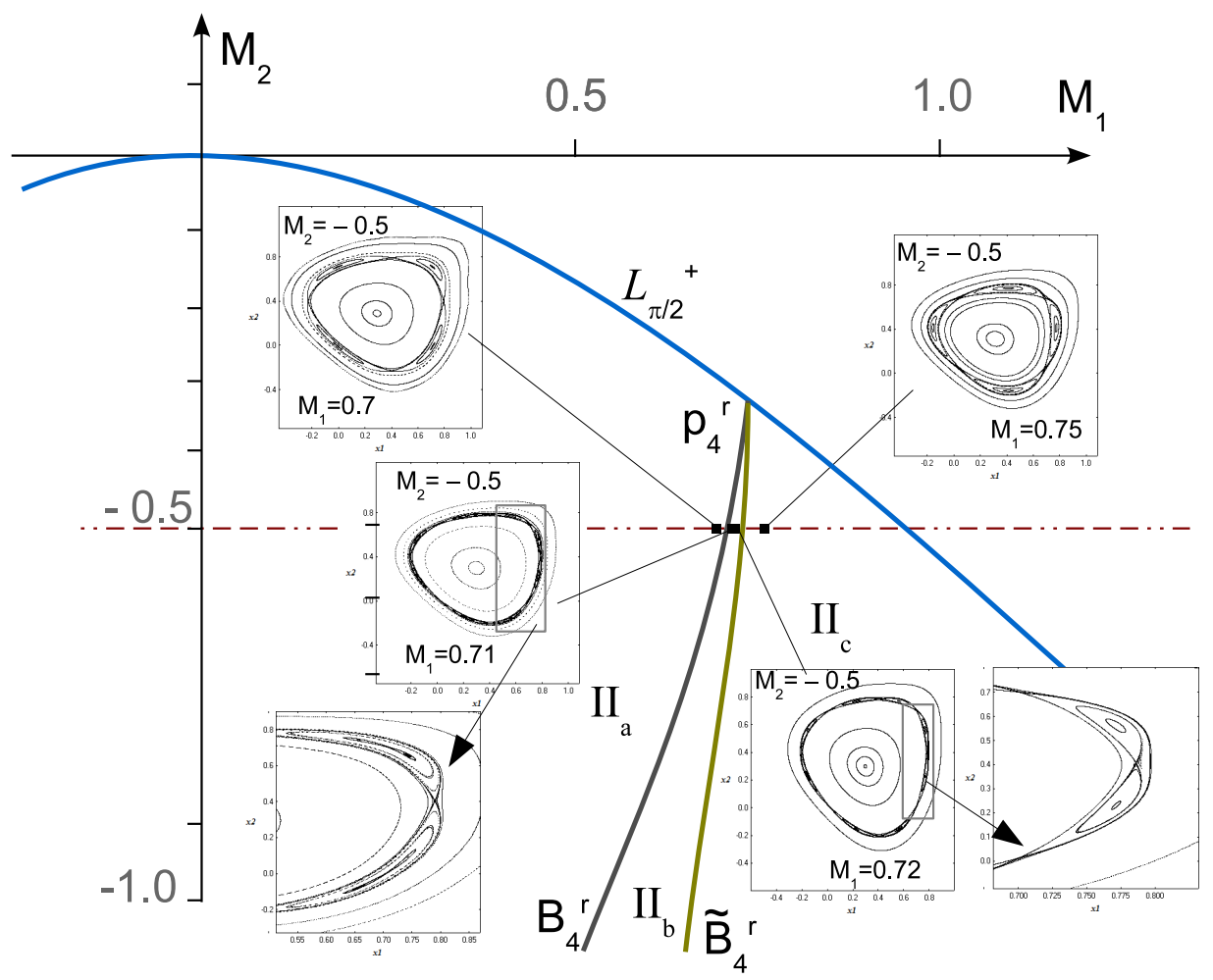

Figure 9: A fragment of bifurcation diagram at crossing curves $B_{4}^{r}$ and $\tilde{B}_{4}^{r}$ when $M_{1}$ changes for a fixed $M_{2}=-0.5$. Effect of thinning of the 1:4 resonance chain is observed and some accompanied pitch-fork bifurcations with 4-periodic orbits are shown.

connected with the reconstruction of a saddle 4-periodic orbit (as at the transition between the domains II and III from the bifurcation diagram of Figure 111).

In the degenerate case $A=1$, the main bifurcations can be described by means of a two parameter family of the Hamiltonian system

$$
\dot{\zeta}=i \beta \zeta+i(1+\mu) \zeta|\zeta|^{2}+i \zeta^{* 3}+i B_{2}|\zeta|^{4} \zeta+i C|\zeta|^{2} \zeta^{* 3}+O\left(|\zeta|^{7}\right)
$$

where $\beta$ and $\mu$ are parameters, $B_{2}$ and $C$ are real coefficients, see more details in Bir87, GG14, SV09. The typical bifurcation diagram 2 for family (24) is shown in Figure 11. It contains three bifurcation curves which divide the $(\beta, \mu)$ parameter plane into 3 domains. Crossing the curves $L_{1}:\{\beta=0, \mu>0\}$ and $L_{2}:\{\beta=0, \mu<0\}$ corresponds to the reconstruction of nonzero saddle equilibria. Curve $L_{3}$ corresponds to the appearance of 4 nonzero parabolic equilibria (this bifurcation is nondegenerate for the family (24) since it is invariant under rotation by angle $\pi / 2$ ). Note that the trivial equilibrium is a nondegenerate conservative center for $\beta \neq 0$; it is a degenerate conservative center for $(\beta, \mu) \in L_{2}$ and a saddle with 8 separatrices for $(\beta, \mu) \in L_{1}$.

Concerning map $\mathbf{C}_{-}^{1}$, the main elements of the bifurcation diagram related to 1:4 resonance are shown in Figure 12 , Here the 4 main bifurcation curves, $L_{\pi / 2}^{-}, L_{4}^{1}, L_{4}^{2}$ and $L_{4}^{3}$, are shown in the $\left(M_{1}, M_{2}\right)$-parameter plane. The equation for $L_{\pi / 2}^{-}$is given by (23), the remaining curves have the following equations

$$
\begin{aligned}
& L_{4}^{1,2}: M_{1}= \pm \frac{2}{3 \sqrt{3}}\left(1+M_{2}\right)^{3 / 2}, M_{2}>1 / 3, \\
& L_{4}^{3}: M_{1}= \pm \frac{2}{3 \sqrt{3}}\left(2+M_{2}\right) \sqrt{M_{2}-1},
\end{aligned}
$$

Curves (25) correspond to nondegenerate parabolic bifurcations for $R$-symmetric 4-periodic orbits. The birth of a pair of saddle and elliptic 4-periodic orbits occurs at upward crossing these curves. Curves $L_{4}^{1}$ and $L_{4}^{2}$ are

\footnotetext{
${ }^{2}$ Note that the nondegeneracy conditions in this case include also the inequality $B_{2} \neq C$, Bir87.
} 


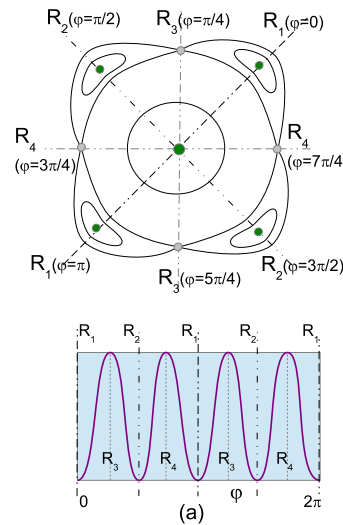

(a)
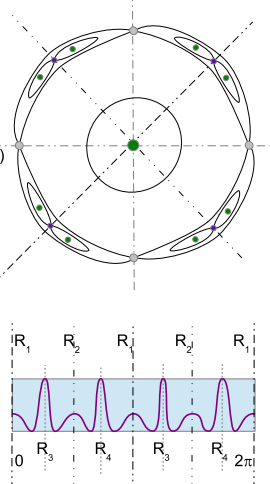

(b)
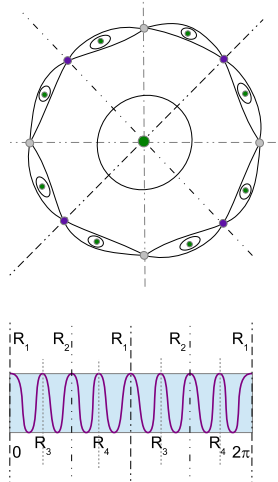

(c)
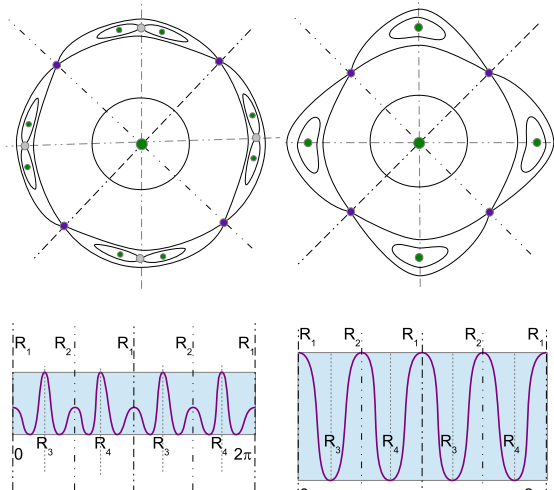

(d)

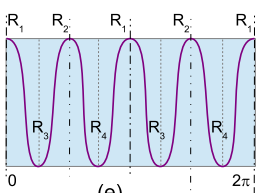

(e)

Figure 10: The main steps of bifurcations inside the 1:4 resonance chain (upper plots), and the corresponding evolution of the appropriate cyclic potential function (lower plots) for the Hamiltonian flow (22) when $\mu$ changes and $B_{1} \beta<0$ is fixed, related to the cases (a) $M_{1}=0.7$, (b) $M_{1}=0.71$, (c) a critical value $0.71<M_{1}<0.72$ corresponding to the homo/heteroclinic zone, (d) $M_{1}=0.72$, (e) $M_{1}=0.75$. Here, $R:\{x=y\}$ and $R^{*}:\{x=-y\}$ are the lines of fixed points of the corresponding involutions. In the lower plots for the potential function, we need to identify the left and rights hand sides $(0$ and $2 \pi)$ of the bands.

(quadratically) tangent to curve $L_{\pi / 2}^{-}$at the points $P^{ \pm}$, see Figure 12, In principle, bifurcations of map $\mathbf{C}_{-}^{1}$, when $M_{1}$ and $M_{2}$ vary near $P^{ \pm}$, look similar to the bifurcations in the flow normal form (24), compare Figures 11 and 12 (in the latter figure, such bifurcations are displayed for the case of point $P^{+}$). The presence of curve $L_{4}^{3}$ shows that the structure of 1:4 resonance in the case of map $\mathbf{C}_{-}^{1}$ is not trivial that can be imagined from Figure 12, where the main elements of the corresponding bifurcation diagram are shown.

\section{References}

[Arn96] V.I. Arnold. Geometrical Methods in the Theory of Ordinary Differential Equations. Springer; 2nd edition, 1996.

[Bir35] G.D. Birkhoff. Nouvelles recherches sur les systèmes dynamiques, Memoriae Pont. Acad. Sci. Novi Lyncaei, 1:85-216, 1935.

[Bir87] V. S. Biragov. Bifurcations in a two-parameter family of conservative mappings that are close to the Hénon mapping. (Russian) Translated in Selecta Math. Soviet. 9(3):273-282, 1990. Methods of the qualitative theory of differential equations (Russian), 10-24, 1987.

[DGG15] A. Delshams, M. Gonchenko, S. Gonchenko. On dynamics and bifurcations of area-preserving maps with homoclinic tangencies. Nonlinearity, 28(9):3027-3071, 2015.

[DGGLS13] A. Delshams, S.V. Gonchenko, V.S. Gonchenko, J.T. Lázaro, O.V. Sten'kin. Abundance of attracting, repelling and elliptic orbits in two-dimensional reversible maps. Nonlinearity, 26(1):1-33, 2013.

[DM00] H.R. Dullin, J.D. Meiss. Generalized Hénon maps: the cubic diffeomorphisms of the plane. Phys. D, 143:262$289,2000$.

[DN05] T. Downarrowicz, S. Newhouse. Symbolic extensions and smooth dynamical systems, Invent. Math., 160:453499, 2005.

[GG09] M. Gonchenko, S. Gonchenko. On cascades of elliptic periodic orbits for area-preserving maps close to a map with a homoclinic tangency, Regul. Chaotic Dyn., 14(1): 116-136, 2009

[GG14] V. Gelfreich, N. Gelfreich. Unique normal forms near a degenerate elliptic point in two-parametric families of area-preserving maps. Nonlinearity, 27(7):1191-1245, 2014. 


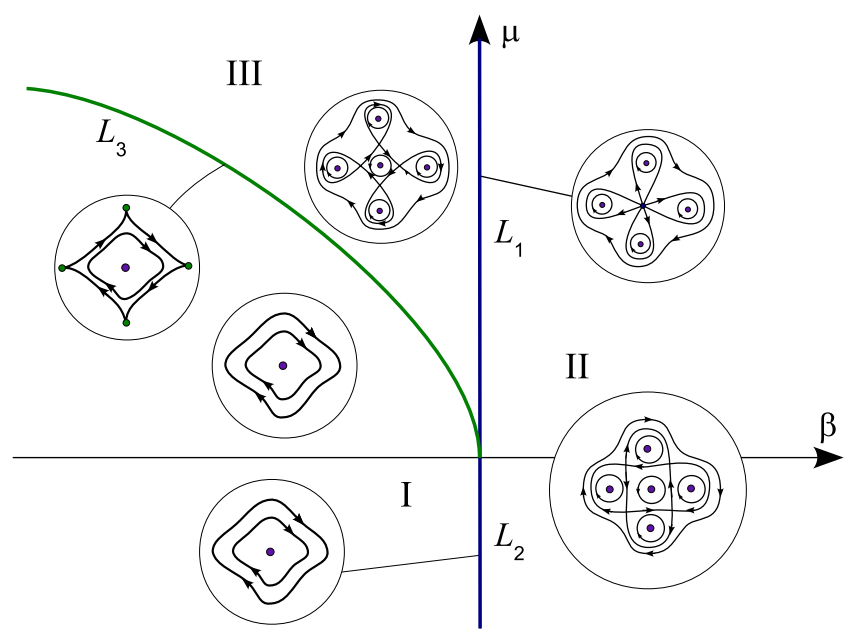

Figure 11: Main elements of bifurcation diagram in the case $A=1$.

[GK88] S.V. Gonchenko, Yu.A. Komlev. Bifurcations and chaos in cubic maps of the plane, Methods of the Qualitative Theory of Differential Equations: Gorky State Univ., 33-40, 1988 (Russian).

[GLRT14] S.V. Gonchenko, J.S.W. Lamb, I. Rios, D. Turaev. Attractors and repellers near generic elliptic points of reversible maps.- Dokl. Math., 89(1):65-67, 2014.

[Gon85] S.V.Gonchenko. On a two parameter family of systems close to a system with a nontransversal Poincaré homoclinic curve. I. Methods of Qualitative Theory of Differential Equations; editor: E.A.Leontovich; Gorky St. Univ., 55-72, 1985 // [English transl. in: Selecta Math. Sovietica,10, 1990].

[Gon05] M.S. Gonchenko. On the structure of 1:4 resonances in Hénon maps, Internat. J. Bifur. Chaos Appl. Sci. Engrg, 15(11):3653-3660, 2005.

[GS73] N. Gavrilov, L. Shilnikov. On three-dimensional systems close to systems with a structurally unstable homoclinic curve: I, Math.USSR-Sb., 17:467-485, 1972; II, Math.USSR-Sb., 19:139-156, 1973.

[GS97] S.V. Gonchenko, L.P. Shilnikov. On two-dimensional analytic area-preserving diffeomorphisms with infinitely many stable elliptic periodic points. Regul. Chaotic Dyn., 2(3/4):106-123, 1997.

[GS00] S.V.Gonchenko, L.P.Shilnikov. On two-dimensional area-preserving diffeomorphisms with infinitely many elliptic islands. J. Statist. Phys., 101(1/2):321-356, 2000.

[GST93] S.V. Gonchenko, L.P. Shilnikov, D.V. Turaev. Dynamical phenomena in systems with structurally unstable Poincaré homoclinic orbits, Russian Acad. Sci. Dokl. Math., 47(3):410-415, 1993.

[GST96] S.V. Gonchenko, L.P. Shilnikov, D.V. Turaev. Dynamical phenomena in systems with structurally unstable Poincaré homoclinic orbits, Chaos 6(1):15-31, 1996.

[GST97] S.V. Gonchenko, L.P. Shilnikov, D.V. Turaev. On Newhouse regions of two-dimensional diffeomorphisms close to a diffeomorphism with a nontransversal heteroclinic cycle, Proc. Steklov Inst. Math., 216:7-118, 1997.

[GST99] S.V. Gonchenko, L.P. Shilnikov, D.V. Turaev. Homoclinic tangencies of an arbitrary order in Newhouse domains, Itogi Nauki Tekh., Ser. Sovrem. Mat. Prilozh., 67:69-128, 1999 [English translation in J. Math. Sci., 105:1738-1778, 2001].

[GST07] S.V. Gonchenko, L.P. Shilnikov, D.V. Turaev. Homoclinic tangencies of arbitrarily high orders in conservative and dissipative two-dimensional maps, Nonlinearity 20:241-275, 2007. 


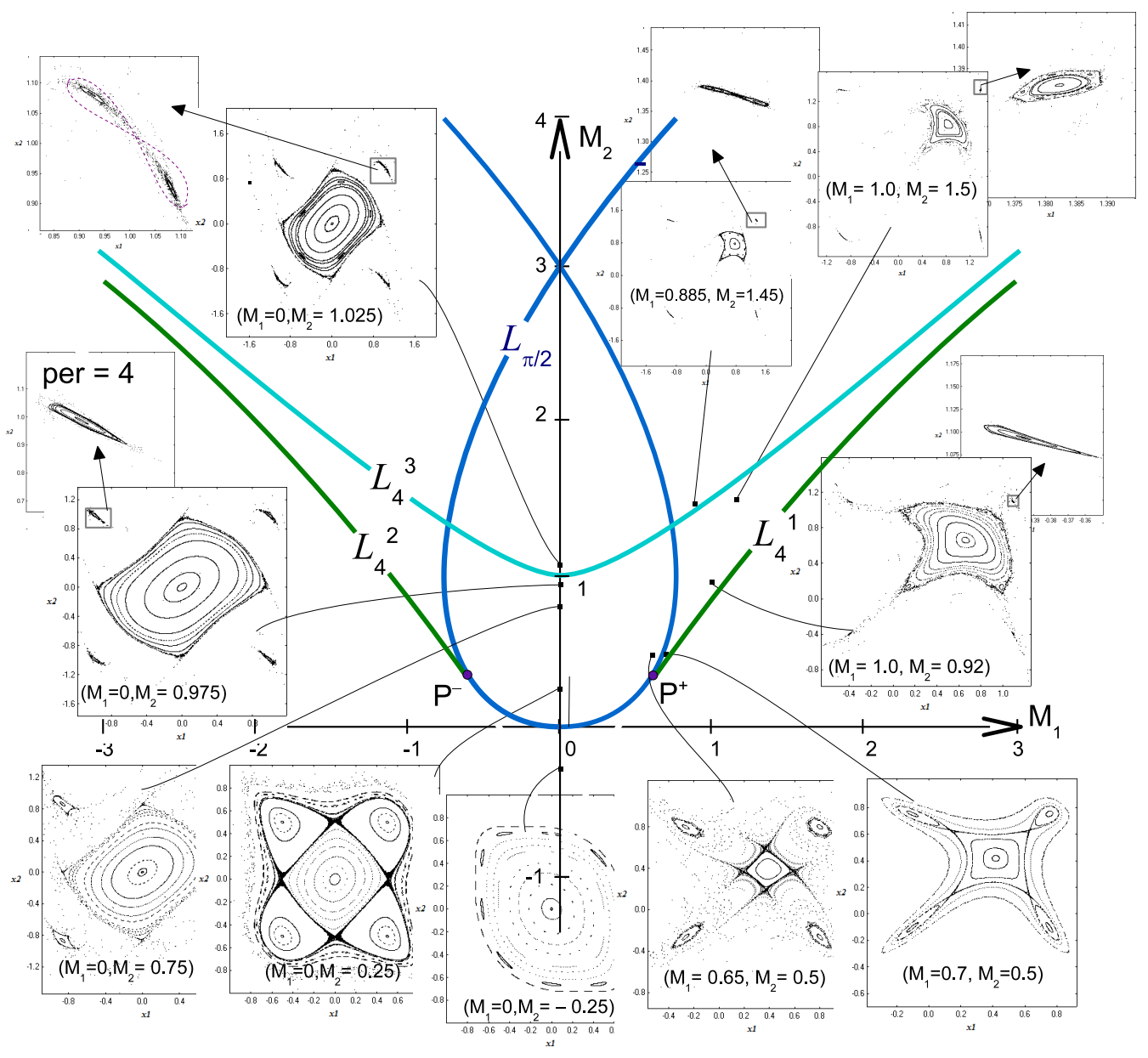

Figure 12: Some elements of the bifurcation diagram for 1:4 resonance in the case of map $\mathbf{C}_{-}^{1}$.

[GSV13] S.V. Gonchenko, C. Simó, A. Vieiro. Richness of dynamics and global bifurcations in systems with a homoclinic figure-eight // Nonlinearity, 26(3):621-678, 2013.

[Kal00] V.Kaloshin, Generic diffeomorphisms with superexponential growth of number of periodic orbits, Commun. Math. Phys. 211: 253-271, 2000.

[LS04] J.S.W. Lamb, O.V. Stenkin. Newhouse regions for reversible systems with infinitely many stable, unstable and elliptic periodic orbits, Nonlinearity, 17:1217-1244, 2004.

[Mos56] J. Moser. The analytic invariants of an area-preserving mapping near a hyperbolic fixed point. Comm. Pure Appl.Math., 9:673-692, 1956.

[Poi90] H. Poincaré. Sur le problème des trois corps et les équations de la dynamique, Acta Math. 13:1-270, 1890.

[Poi99] H. Poincaré. Les méthodes nouvelles de la mécanique céleste, Tome III, Gauthier-Villars, 1899.

[RT99] V. Rom-Kedar, D. Turaev. Big islands in dispersing billiard-like potencials. Phys. D, 130(3-4):187-210, 1999.

[Shi67] L.P. Shilnikov. On a Poincaré-Birkhoff problem, Math. USSR. Sb. 3:353-371, 1967.

[Sma65] S. Smale. Diffeomorphisms with many periodic points, Differential and Combinatorial Topology, Princeton Univ. Press, 63-80, 1965. 
[SSTC98] L.P. Shilnikov, A.L. Shilnikov, D.V. Turaev, L.O. Chua. Methods of qualitative theory in nonlinear dynamics, Part I. World Scientific, 1998.

[SV09] C. Simó, A. Vieiro. Resonant zones, inner and outer splitting in generic and low order resonances of area preserving maps. Nonlinearity, 22(5):1191-1245, 2009.

[Tat92] J.C. Tatjer. Bifurcations of codimension two near homoclinic tangencies of second order. In Proceedings of the European Conference on Iteration Theory, World Sci. Publishing, Teaneck,NJ. 306-318, 1992.

[TY86] L. Tedeschini-Lalli, J.A. Yorke. How often do simple dynamical processes have infinitely many coexisting sinks? Commun. Math. Phys., 106:635-657, 1986. 